\title{
The influence of the growing flexible shell on the molecular behavior of hybrid dendrimers
}

Sergey A. Milenin ${ }^{a^{*}}$, Georgy V. Cherkaev a, Nina V. Demchenko a, Elena S. Serkova ${ }^{b}$, Irina Yu. Krasnova ${ }^{b}$, Elizaveta V. Selezneva ${ }^{a}$, Mikhail I. Buzin ${ }^{\text {b }}$, Artem V. Bakirov ${ }^{\text {a,c }}$, Viktor G. Vasil'ev ${ }^{\text {b }}$, Zinaida B. Shifrina ${ }^{\text {b, }}$ Sergey N. Chvalun ${ }^{c}$, Aziz M. Muzafarov ${ }^{a}$

${ }^{a}$ N.S. Enikolopov Institute of Synthetic Polymeric Materials, Russian Academy of Sciences, 70 Profsouznaya St., Moscow, 117393, Russia

${ }^{b}$ A.N. Nesmeyanov Institute of Organoelement Compounds, Russian Academy of Sciences, 28 Vavilov St., Moscow, 119991 Russia

c National Research Center "Kurchatov Institute”, Akademika Kurchatova pl., 1, 123182 Moscow, Russia 


\section{Materials and methods}

All the starting materials were purchased from Acros and Sigma Aldrich Company. Solvents were dried and purified according to standard procedures, $\mathrm{MeCN}$ was purchased from PanReac and used without further purification.

${ }^{1} \mathrm{H},{ }^{13} \mathrm{C},{ }^{29} \mathrm{Si}$ and $\mathrm{HMBC}$ NMR spectra were recorded using a Bruker Avance $300 \mathrm{NMR}$ spectrometer in $\mathrm{CDCl}_{3}$ and $\mathrm{C}_{3} \mathrm{D}_{6} \mathrm{O}$, chemical shifts are referenced to residual chloroform $\left(7.26 \mathrm{ppm},{ }^{1} \mathrm{H}\right)$. Chemical shifts are reported in ppm, multiplicities are indicated by s (singlet), d (doublet), t (triplet), q (quartet), p (pentet), h (hextet), m (multiplet) and br (broad).

High resolution mass spectra (HRMS) were registered on a Bruker Daltonics micrOTOF-Q II instrument using electrospray ionization (ESI). The measurements were acquired in a positive ion mode with the following parameters: interface capillary voltage $4500 \mathrm{~V}$; mass range from m/z 50 to 3000; external calibration (Electrospray Calibrant Solution, Fluka); nebulizer pressure 0.4 Bar; flow rate $3 \mu \mathrm{L} / \mathrm{min}$; nitrogen was applied as a dry gas $(6 \mathrm{~L} / \mathrm{min})$; interface temperature was set at $180^{\circ} \mathrm{C}$. Samples were introduced into the mass spectrometer chamber via syringe injection, compounds were dissolved in acetonitrile (LC-MS grade, Panreac). Certain experiments were performed using dopants to the samples, e.g. HCOONa, $0.05 \% \mathrm{HCl}$ solution in water (Merck Milli-Q grade), $0.5 \% \mathrm{NEt}_{3}$ solution in acetonitrile.

GPC analysis was performed on the "Shimadzu" (Japan, Germany), the detector - refractometer RID - $20 \AA$, the column -

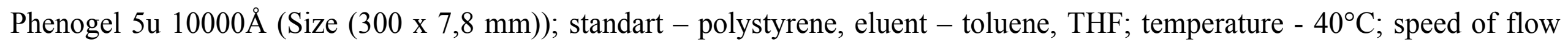
$1 \mathrm{ml} / \mathrm{sec}$.

The preparative chromatographic system consisted of a high-pressure isocratic pump (SHIMADZU, LC-20AT), a RIDK102 refractometric detector (Czechia), and $300 \times 21.2 \mathrm{~mm}$ Phenomenex preparative columns (USA)packed with the Phenogel sorbent (particle size $10 \mu \mathrm{m}$ ). Depending on the molecular masses of the components of the mixtures being separated, columns with pore sizes of 103,104 , and $105 \AA$ were used and THF was used as the eluent. 
Synthesis carbosilane monodendrons:

$\mathbf{D}_{1} \mathbf{S i}_{3} \mathbf{A l l}_{4}(\mathbf{C l})$ (7) Synthesized from $2 \mathrm{~g}(0.01 \mathrm{~mol})$ of 3-chloropropyldiallylmethylsilane. The product was a colorless liquid and was used without further purification. Yield was about $4.2 \mathrm{~g}(90 \%) .{ }^{1} \mathrm{H}$ NMR $\left(\mathrm{CDCl}_{3}\right): \delta 5.76\left(\mathrm{~m}, 4 \mathrm{H}, \mathrm{CH}_{2}=\mathrm{CH}_{-}-\mathrm{CH}_{2}-\mathrm{Si}\right), 4.85$ (m, 8H, $\left.\underline{\mathrm{CH}}_{2}=\mathrm{CH}-\mathrm{CH}_{2}-\mathrm{Si}\right), 3.49$ (t, 2H, Cl- $\left.\underline{\mathrm{H}}_{2}-\mathrm{CH}_{2}-\mathrm{CH}_{2}-\mathrm{Si}\right), 1.74\left(\mathrm{~m}, 2 \mathrm{H}, \mathrm{Cl}-\mathrm{CH}_{2}-\mathrm{CH}_{2}-\mathrm{CH}_{2}-\mathrm{Si}\right), 1.53\left(\mathrm{~m}, 8 \mathrm{H}, \mathrm{CH}{ }_{2}=\mathrm{CH}-\mathrm{CH} 2-\mathrm{Si}\right)$, $1.33\left(\mathrm{~m}, 4 \mathrm{H}, \mathrm{Si}-\mathrm{CH}_{2}-\underline{\mathrm{CH}}_{2}-\mathrm{CH}_{2}-\mathrm{Si}\right), 0.59\left(\mathrm{~m}, 10 \mathrm{H},-\mathrm{CH}_{2}-\mathrm{Si}\right),-0.02\left(\mathrm{~s}, 3 \mathrm{H}, \mathrm{Si}-\mathrm{CH}_{3}\right),-0.05\left(\mathrm{~s}, 6 \mathrm{H}, \mathrm{Si}_{-} \mathrm{CH}_{3}\right) ;{ }^{13} \mathrm{C} \mathrm{NMR}\left(\mathrm{CDCl}_{3}\right): \delta 13 \mathrm{C}$ $\operatorname{NMR}(75 \mathrm{MHz}, \mathrm{CDCl}) \delta 134.77,113.06,48.03,27.72,21.45,18.50,18.14,17.90,11.57,-5.19,-5.75 .{ }^{29} \mathrm{Si}^{\mathrm{NMR}}\left(\mathrm{CDCl}_{3}\right): \delta 2.09$, 0.21 , GPC: One peak - $500 \mathrm{~g} / \mathrm{mol}$.

$\mathbf{D}_{2} \mathbf{S i}_{7} \mathbf{B} \mathbf{u}_{\mathbf{8}}(\mathbf{C l})$ (9) Synthesized from $1.53 \mathrm{~g}$ (3.36 mmol) of $\mathrm{D}_{1} \mathrm{Si}_{3} \mathrm{All}_{4}(\mathrm{Cl})(7)$. The product was a colorless liquid and was used without further purification. Yield was about $3.07 \mathrm{~g}(84 \%) .{ }^{1} \mathrm{H}$ NMR $\left(\mathrm{CDCl}_{3}\right): \delta 3.48\left(\mathrm{t}, 2 \mathrm{H}, \mathrm{Cl}_{-}-\mathrm{CH}_{2}-\mathrm{CH}_{2}-\mathrm{CH}_{2}-\mathrm{Si}\right), 1.73(\mathrm{~m}, 2 \mathrm{H}$, Cl- $\mathrm{CH}_{2}-\underline{\mathrm{CH}}_{2}-\mathrm{CH}_{2}-\mathrm{Si}$ ), 1.3 (m, 44H, Si-CH $\left.2-\underline{\mathrm{CH}}_{2}-\mathrm{CH}_{2}-\mathrm{Si}, \mathrm{CH}_{3}-\underline{\mathrm{CH}}_{2}-\mathrm{CH}_{2}-\mathrm{CH}_{2}-\mathrm{Si}\right), 0.88\left(\mathrm{~m}, 24 \mathrm{H}, \mathrm{CH}_{3}-\mathrm{CH}_{2}-\right), 0.52\left(\mathrm{~m}, 42 \mathrm{H},-\mathrm{CH}_{2}-\right.$ $\mathrm{Si}),-0.05$ (s, 3H, $\left.\mathrm{CH}_{3}-\mathrm{Si}\right),-0.09$ (s, 18H, $\left.\mathrm{CH}_{3}-\mathrm{Si}\right) ;{ }^{13} \mathrm{C} \mathrm{NMR}\left(\mathrm{CDCl}_{3}\right): \delta 47.94,27.91,26.69,26.27,19.15,19.07,18.96,18.86$, 18.59, 18.54, 13.78, 11.87, -4.87, -5.01, -5.07.; ${ }^{29} \mathrm{Si} \mathrm{NMR}\left(\mathrm{CDCl}_{3}\right): \delta 2.28,2.03,1.00$. GPC: One peak- $1700 \mathrm{~g} / \mathrm{mol}$.

$\mathbf{D}_{2} \mathbf{S i}_{7} \mathbf{A l l}_{\mathbf{8}}(\mathbf{C l})$ (11) Synthesized from $2.08 \mathrm{~g}(4.57 \mathrm{mmol}) \mathrm{D}_{1} \mathrm{Si}_{3} \mathrm{All}_{4}(\mathrm{Cl})(7)$. The product was a colorless liquid and was used without further purification. Yield was about $3.31 \mathrm{~g}(75 \%) .{ }^{1} \mathrm{H} \mathrm{NMR}\left(\mathrm{CDCl}_{3}\right): \delta 5.76\left(\mathrm{~m}, 8 \mathrm{H}, \mathrm{CH}_{2}=\mathrm{CH}_{-}-\mathrm{CH}-\mathrm{Si}\right), 4.86(\mathrm{~m}, 16 \mathrm{H}$, $\left.\mathrm{CH}_{2}=\mathrm{CH}-\mathrm{CH}_{2}-\mathrm{Si}\right), 3.49\left(\mathrm{t}, 2 \mathrm{H}, \mathrm{Cl}-\mathrm{CH}_{2}-\mathrm{CH}_{2}-\mathrm{CH}_{2}-\mathrm{Si}\right), 1.74\left(\mathrm{~m}, 2 \mathrm{H}, \mathrm{Cl}-\mathrm{CH}_{2}-\mathrm{CH}_{2}-\mathrm{CH}_{2}-\mathrm{Si}\right), 1.53\left(\mathrm{~m}, 16 \mathrm{H}, \mathrm{CH}=\mathrm{CH}_{2} \underline{\mathrm{H}}_{2}-\mathrm{Si}\right), 1.31$ (m, 12H, Si-CH $\mathrm{CH}_{2}-\mathrm{CH}_{2}-\mathrm{Si}$ ), 0.59 (m, 26H, $-\mathrm{CH}_{2}-\mathrm{Si}$ ), -0.02 (s, 12H, Si-CH ${ }_{3}$ ), -0.04 (s, 3H, Si-CH $)$, -0.08 (s, 6H, Si-CH $)$; GPC: One peak - $1400 \mathrm{~g} / \mathrm{mol}$.

$\mathbf{D}_{3} \mathbf{S i}_{15} \mathbf{B u}_{16}(\mathbf{C l})$ (13) Synthesized from $2.2 \mathrm{~g}$ (3.67 mmol) $\mathrm{D}_{2} \mathrm{Si}_{7} \mathrm{All}_{8}(\mathrm{Cl})(11)$. The product was a colorless liquid and was used without further purification. Yield was about $3.8 \mathrm{~g}(88 \%) .{ }^{1} \mathrm{H} \mathrm{NMR}\left(\mathrm{CDCl}_{3}\right): \delta 3.47\left(\mathrm{t}, 2 \mathrm{H}, \mathrm{Cl}_{-}-\mathrm{CH}_{2}-\mathrm{CH}_{2}-\mathrm{CH}_{2}-\mathrm{Si}\right), 1.74(\mathrm{~m}, 2 \mathrm{H}$, $\left.\mathrm{Cl}-\mathrm{CH}_{2}-\mathrm{CH}_{2}-\mathrm{CH}_{2}-\mathrm{Si}\right), 1.28\left(\mathrm{~m}, 92 \mathrm{H}, \mathrm{Si}-\mathrm{CH}_{2}-\mathrm{CH}_{2}-\mathrm{CH}_{2}-\mathrm{Si}, \mathrm{CH}_{3}-\mathrm{CH}_{2}-\mathrm{CH}_{2}-\mathrm{CH}_{2}-\mathrm{Si}\right), 0.87$ (m, 48H, $\left.\underline{\mathrm{CH}}_{3}-\mathrm{CH}_{2}-\right), 0.5\left(\mathrm{~m}, 90 \mathrm{H}^{-}-\mathrm{CH}_{2}-\right.$ $\mathrm{Si}),-0.06--0.09\left(\mathrm{~m}, 45 \mathrm{H}, \mathrm{CH}_{3}-\mathrm{Si}\right)$; ${ }^{13} \mathrm{C} \mathrm{NMR}\left(\mathrm{CDCl}_{3}\right): \delta 47.94,27.91,26.69,26.27,19.15,19.07,18.96,18.86,18.59,18.54$, 13.78, 11.87, -4.87, -5.01, -5.07; ${ }^{29} \mathrm{Si} \mathrm{NMR}\left(\mathrm{CDCl}_{3}\right): \delta 2.27,2.01,0.98,0.93$. GPC: One peak - 3600 g/mol. 
$\mathbf{D}_{2} \mathbf{S i}_{7} \mathbf{B u}_{\mathbf{8}}\left(\mathbf{N}_{3}\right)$ (10) Synthesized from $2.7 \mathrm{~g}(2.5 \mathrm{mmol}) \mathrm{D}_{2} \mathrm{Si}_{7} \mathrm{Bu}_{8}(\mathrm{Cl})(9)$ and $0.19 \mathrm{~g}(2.86 \mathrm{mmol})$ sodium azide. The product was a colorless liquid and was used without further purification. The reaction lasted for 48 hours until the complete substitution of chlorine. Substitution was $80 \%$ according to NMR. ${ }^{1} \mathrm{H}$ NMR $\left(\mathrm{CDCl}_{3}\right): \delta 3.48\left(\mathrm{t}, 2 \mathrm{H}, \mathrm{Cl}^{-} \mathrm{C}_{2}-\mathrm{CH}_{2}-\mathrm{CH}_{2}-\mathrm{Si}\right), 3.22\left(\mathrm{t}, 2 \mathrm{H}, \mathrm{N}_{3}-\mathrm{CH}_{2}-\right.$ $\mathrm{CH}_{2}-\mathrm{CH}_{2}-\mathrm{Si}$ ), 1.73 (m, 2H, Cl- $\left.\mathrm{CH}_{2}-\mathrm{CH}_{2}-\mathrm{CH}_{2}-\mathrm{Si}\right), 1.58$ (m, 2H, N3- $\mathrm{CH}_{2}-\mathrm{CH}_{2}-\mathrm{CH}_{2}-\mathrm{Si}$ ), 1.3 (m, 44H, Si-CH ${ }_{2}-\mathrm{CH}_{2}-\mathrm{CH}_{2}-\mathrm{Si}, \mathrm{CH}_{3}-$ $\left.\mathrm{C}_{2}-\underline{\mathrm{C}}_{2}-\mathrm{CH}_{2}-\mathrm{Si}\right), 0.88\left(\mathrm{~m}, 24 \mathrm{H}, \mathrm{C}_{3}-\mathrm{CH}_{2}-\right), 0.52\left(\mathrm{~m}, 42 \mathrm{H},-\mathrm{CH}_{2}-\mathrm{Si}\right),-0.05$ (s, 3H, $\left.\mathrm{CH}_{3}-\mathrm{Si}\right),-0.09$ (s, $\left.18 \mathrm{H}, \mathrm{CH}_{3}-\mathrm{Si}\right) ;{ }^{29} \mathrm{Si} \mathrm{NMR}$ $\left(\mathrm{CDCl}_{3}\right): \delta{ }^{29} \mathrm{Si} \mathrm{NMR}(60 \mathrm{MHz}, \mathrm{CDCl} 3) \delta 2.28,2.06,2.04,1.01$. GPC: One peak $-1500 \mathrm{~g} / \mathrm{mol}$.

$\mathbf{D}_{3} \mathbf{S i}_{15} \mathbf{B u}_{16}\left(\mathbf{N}_{3}\right)$ (14) Synthesized from $3 \mathrm{~g}(2.76 \mathrm{mmol})$ D2Si7Bu8(Cl) (9) and $0.2 \mathrm{~g}(3.2 \mathrm{mmol})$ sodium azide. The product was a colorless liquid and was used without further purification. The reaction lasted for 72 hours until the complete substitution of chlorine. Substitution was $60 \%$ according to NMR. ${ }^{1} \mathrm{H}$ NMR $\left(\mathrm{CDCl}_{3}\right): \delta 3.47$ (t, $\left.2 \mathrm{H}, \mathrm{Cl}-\mathrm{C}_{2}-\mathrm{CH}_{2}-\mathrm{CH}_{2}-\mathrm{Si}\right), 3.22\left(\mathrm{t}, 2 \mathrm{H}, \mathrm{N}_{3}-\mathrm{C}_{2}-\right.$ $\mathrm{CH}_{2}-\mathrm{CH}_{2}-\mathrm{Si}$ ), 1.74 (m, 2H, Cl- $\left.\mathrm{CH}_{2}-\mathrm{C}_{2}-\mathrm{CH}_{2}-\mathrm{Si}\right), 1.58$ (m, 2H, N${ }_{3}-\mathrm{CH}_{2}-\mathrm{C}_{2}-\mathrm{CH}_{2}-\mathrm{Si}$ ), 1.28 (m, 92H, Si-CH $\mathrm{CH}_{2}-\mathrm{CH}_{2}-\mathrm{CH}_{2}-\mathrm{Si}_{2} \mathrm{CH}_{3}-$ $\mathrm{C}_{2}-\mathrm{CH}_{2}-\mathrm{CH}_{2}-\mathrm{Si}$ ), 0.87 (m, 48H, $\underline{\mathrm{H}}_{3}-\mathrm{CH}_{2}-$ ), 0.5 (m, 90H, $-\mathrm{CH}_{2}-\mathrm{Si}$ ), -0.06 - -0.09 (m, 45H, $\mathrm{CH}_{3}-\mathrm{Si}$ ); GPC: One peak - $3600 \mathrm{~g} / \mathrm{mol}$. 
2. ${ }^{1} \mathrm{H},{ }^{13} \mathrm{C}$ and ${ }^{29} \mathrm{Si}, \mathrm{HSQC}$ and HMBC NMR spectra and GPC curves of $7-14$, and final dendrimers 
${ }^{1} \mathrm{H}$ NMR (300 MHz, $\mathrm{CDCl}_{3}$ ) of compound 7

sam825.111_D1Si3All4(Cl).1.fid

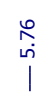

釆

$\underset{\substack{g \\ j}}{j}$

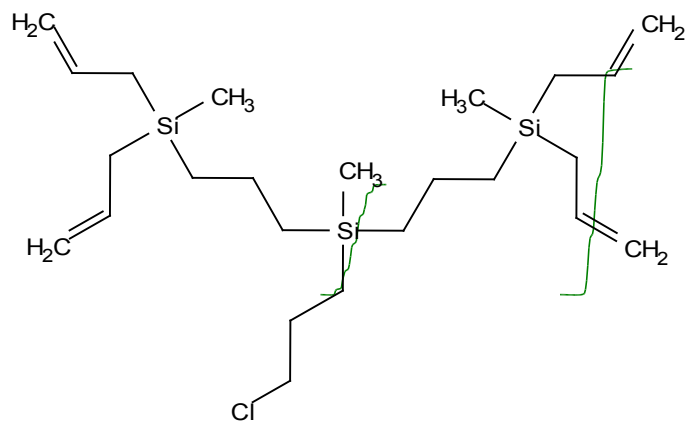

$\stackrel{*}{2}$

参

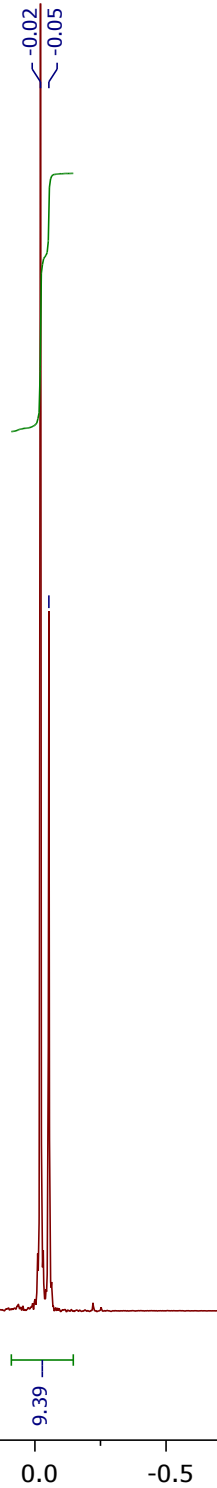


ST

${ }^{13} \mathrm{C}$ NMR (75.5 MHz, $\mathrm{CDCl}_{3}$ of compound 7
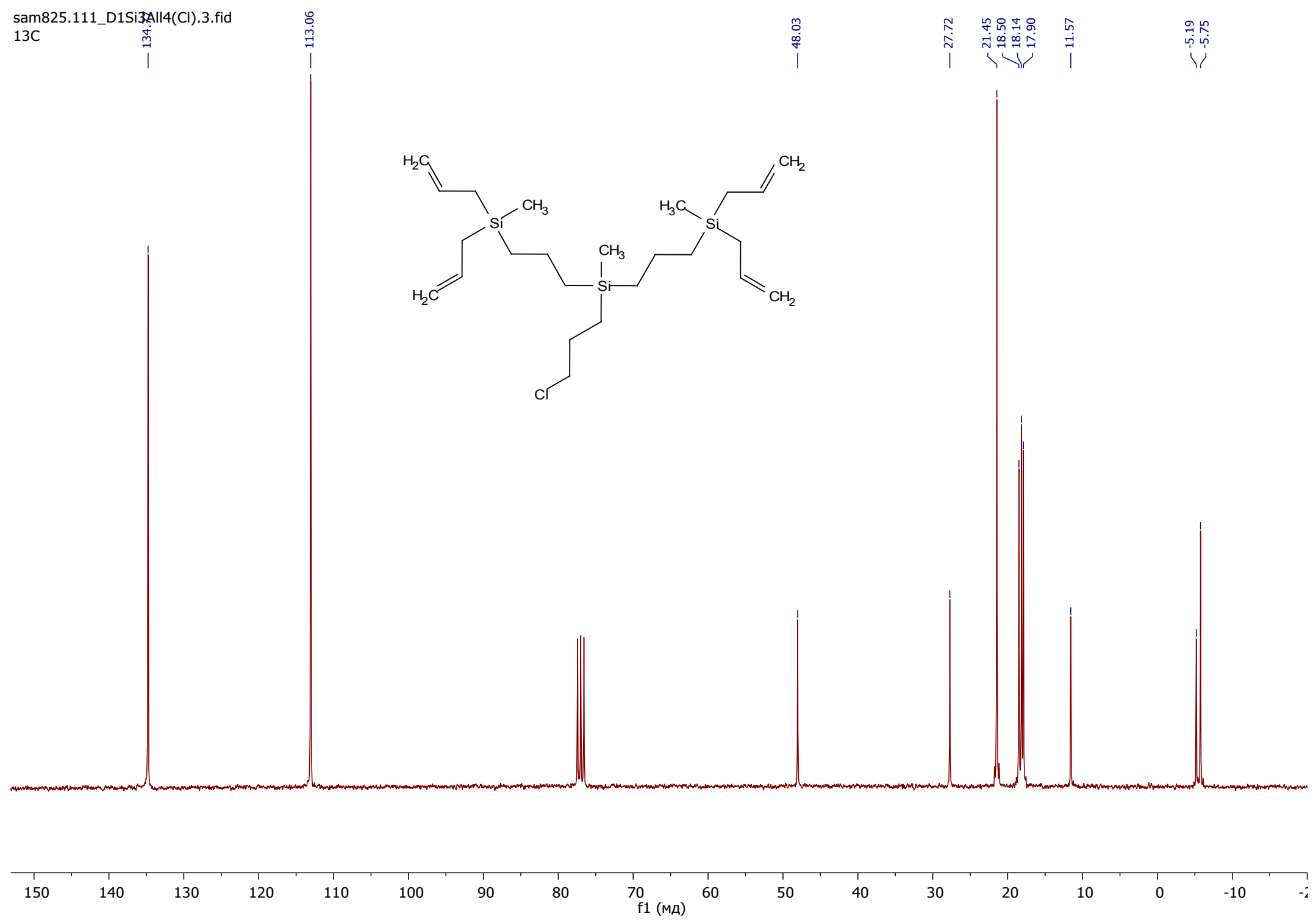
${ }^{29} \mathrm{Si}$ NMR (59.6 MHz, $\mathrm{CDCl}_{3}$ ) of compound 7

sam825.111_D1Si3All4(Cl).2903.fid

29Si_P0 $=$ pi $/ 8 \mathrm{~d} 1=3 \mathrm{~J}=20$
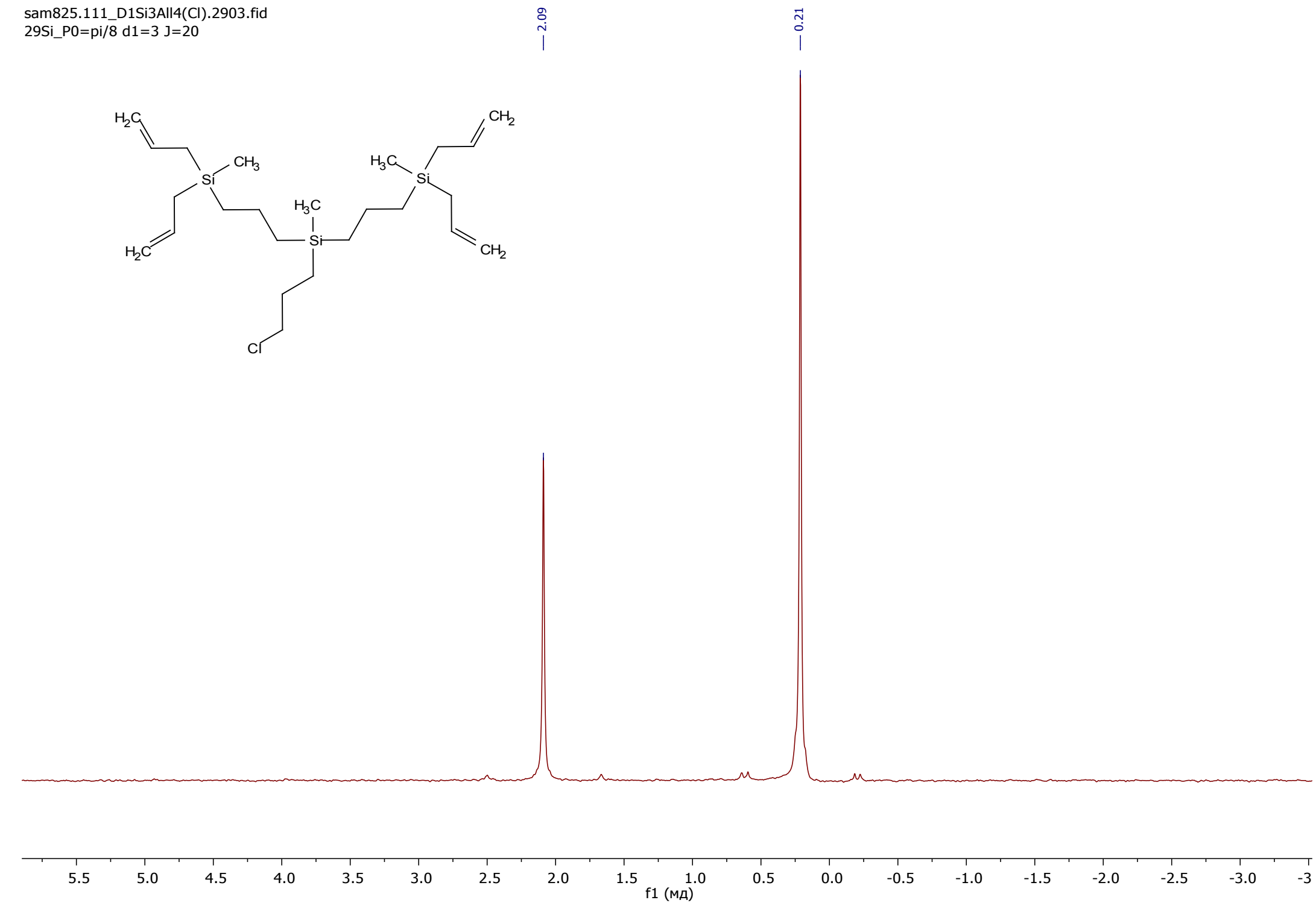
GPC curve of compound 7

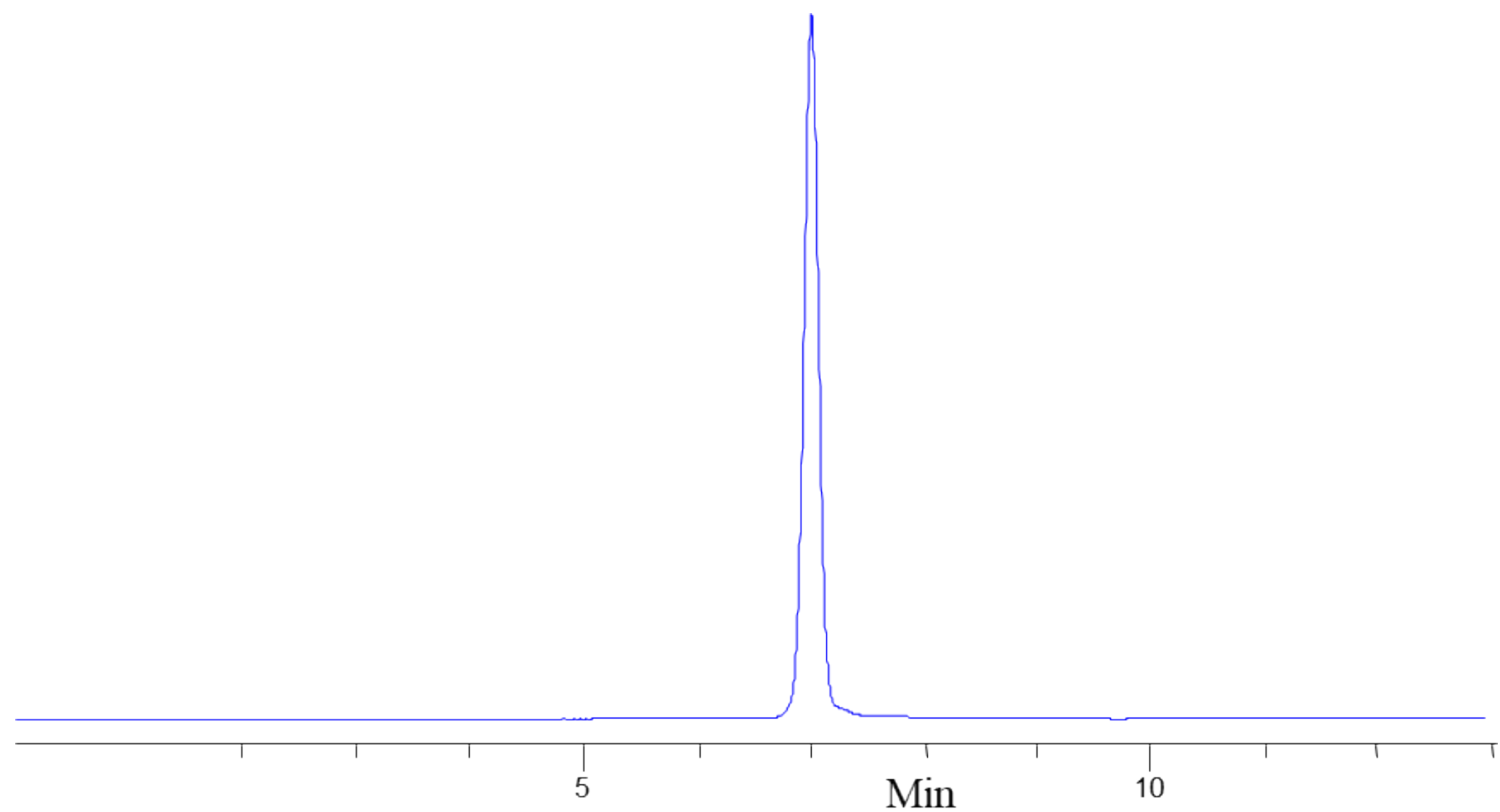


${ }^{1} \mathrm{H}$ NMR $\left(300 \mathrm{MHz}, \mathrm{CDCl}_{3}\right)$ of compound 9

sam861.001_D2Si7Bu8(Cl).1.fid

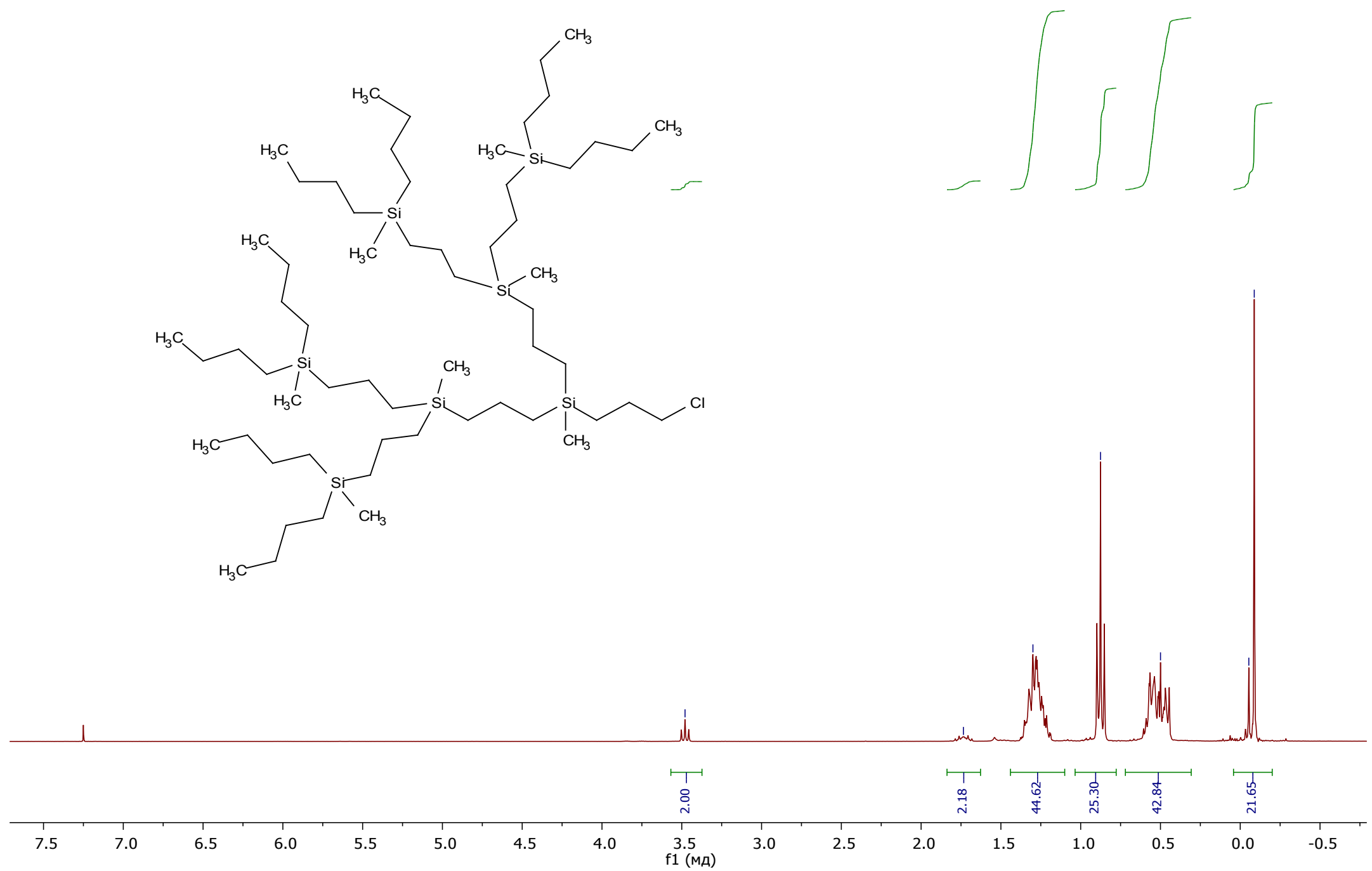


${ }^{13} \mathrm{C}$ NMR (75.5 MHz, $\mathrm{CDCl}_{3}$ of compound 9

sam861.001_D2Si7Bu8(Cl).3.fid

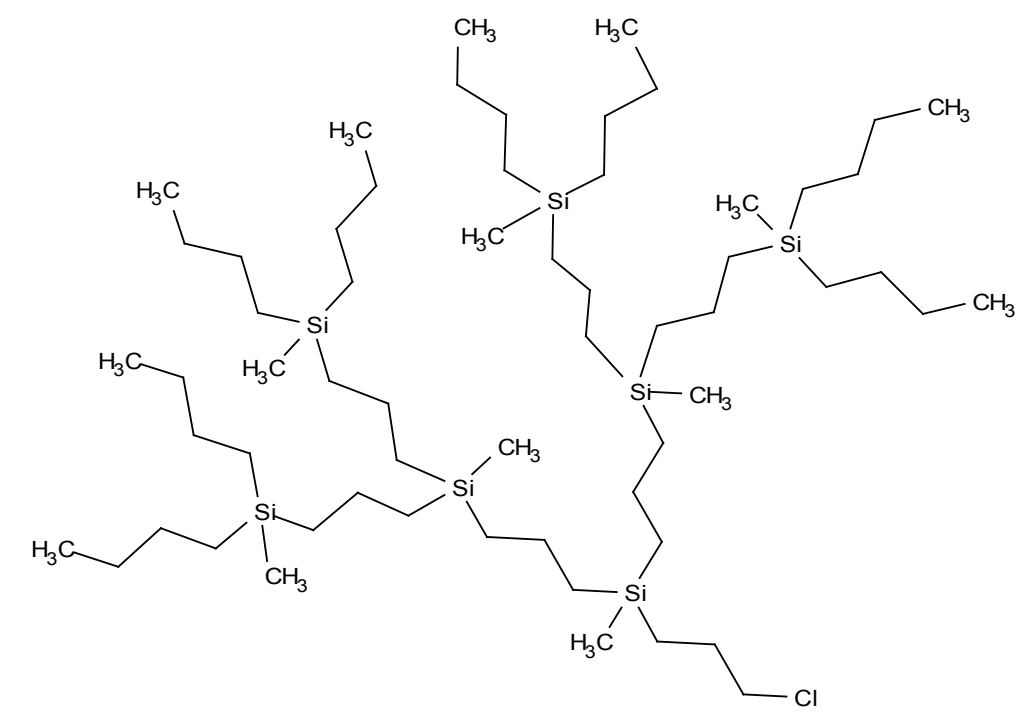

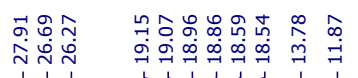

1)
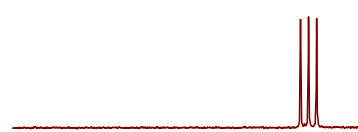
${ }^{29} \mathrm{Si}$ NMR (59.6 MHz, $\mathrm{CDCl}_{3}$ ) of compound 9

sam861.001_D2Si7Bu8(Cl).2903.fid 29Si_P0 $=\mathrm{pi} / 8 \mathrm{~d} 1=3 \mathrm{~J}=20$

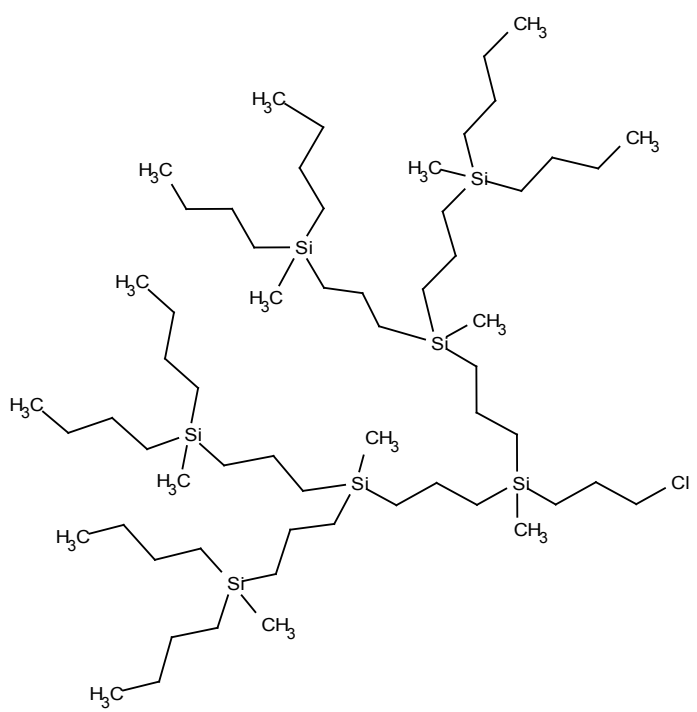


GPC curve of compound 9

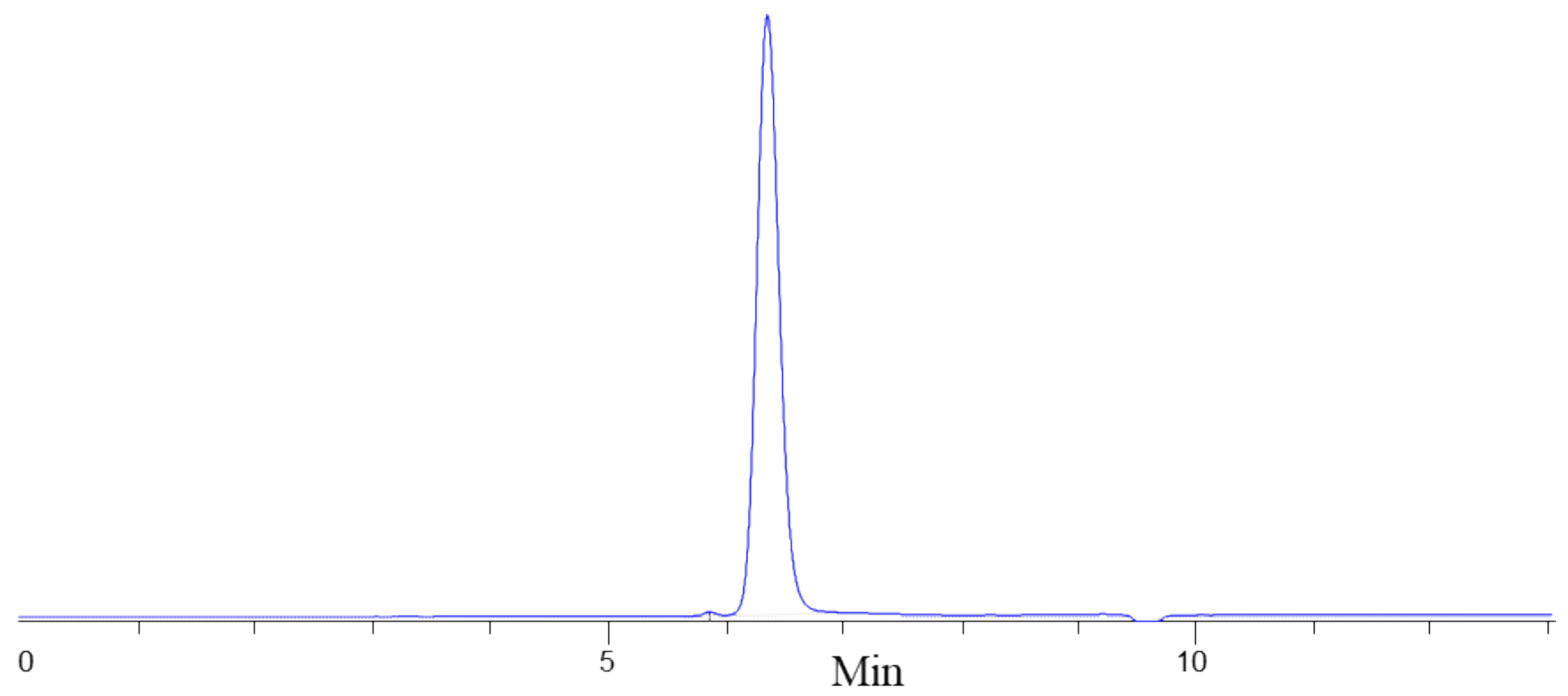


${ }^{1} \mathrm{H}$ NMR $\left(300 \mathrm{MHz}, \mathrm{CDCl}_{3}\right.$ ) of compound 11

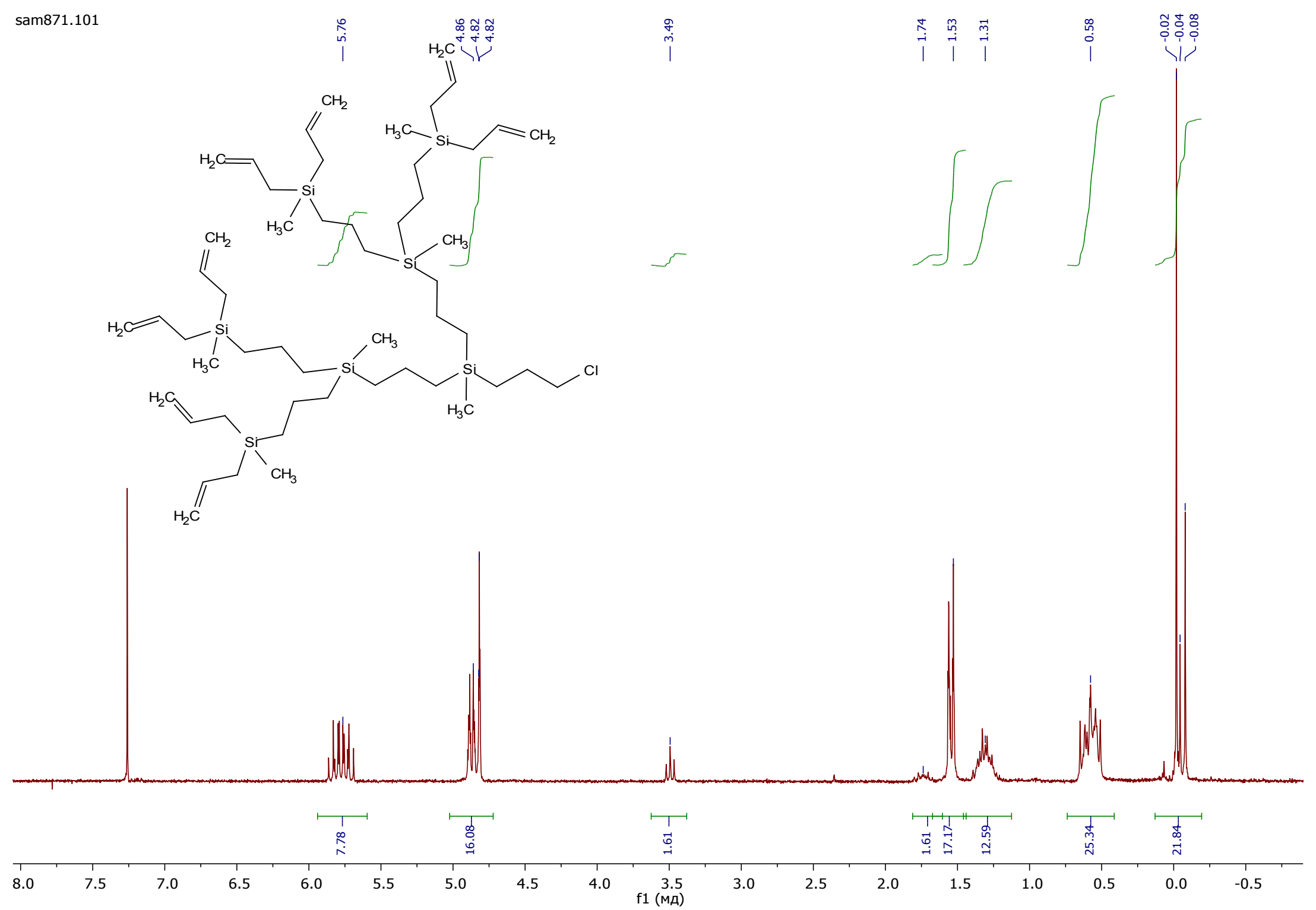


GPC curve of compound 11

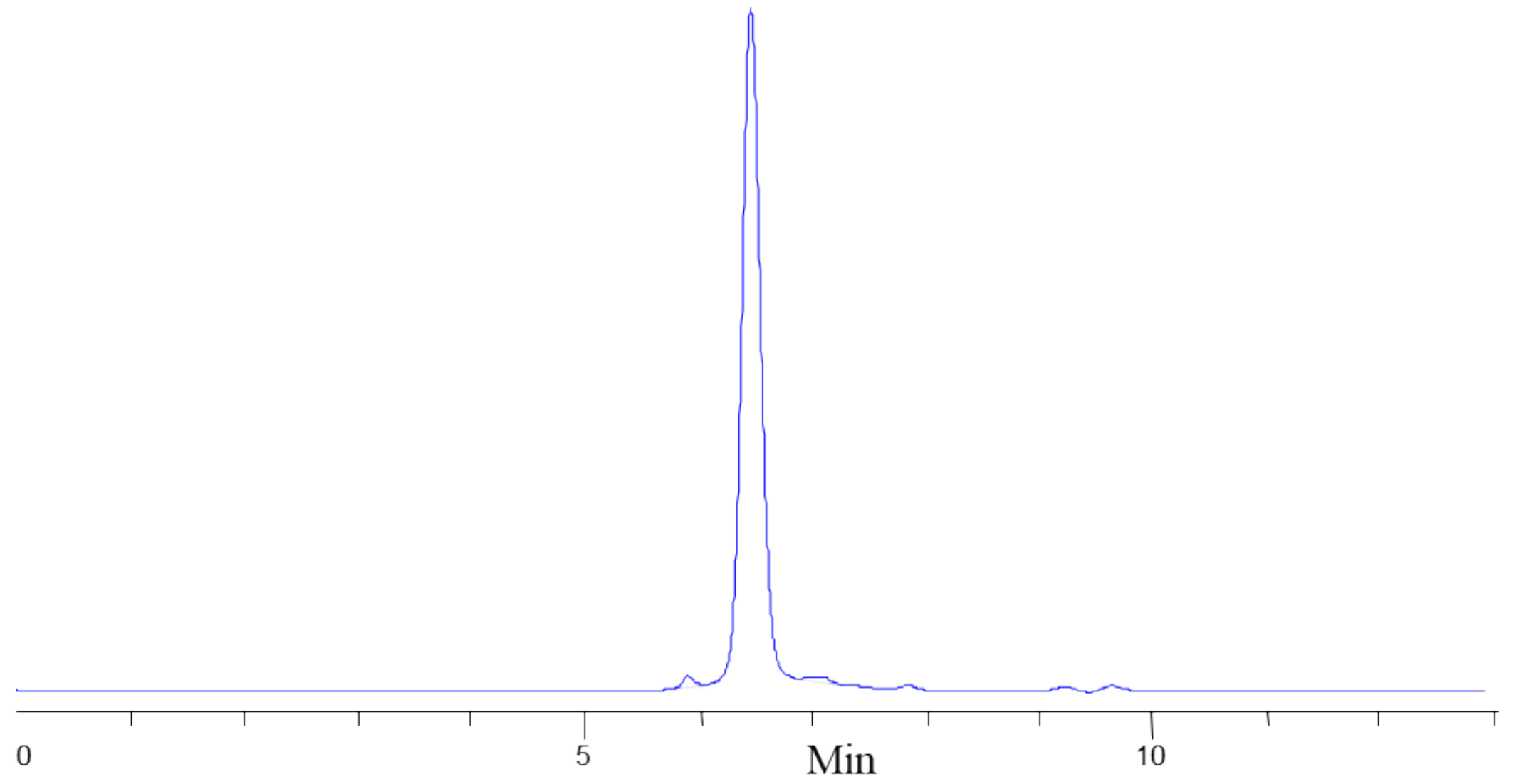


${ }^{1} \mathrm{H}$ NMR (300 MHz, $\mathrm{CDCl}_{3}$ ) of compound 13

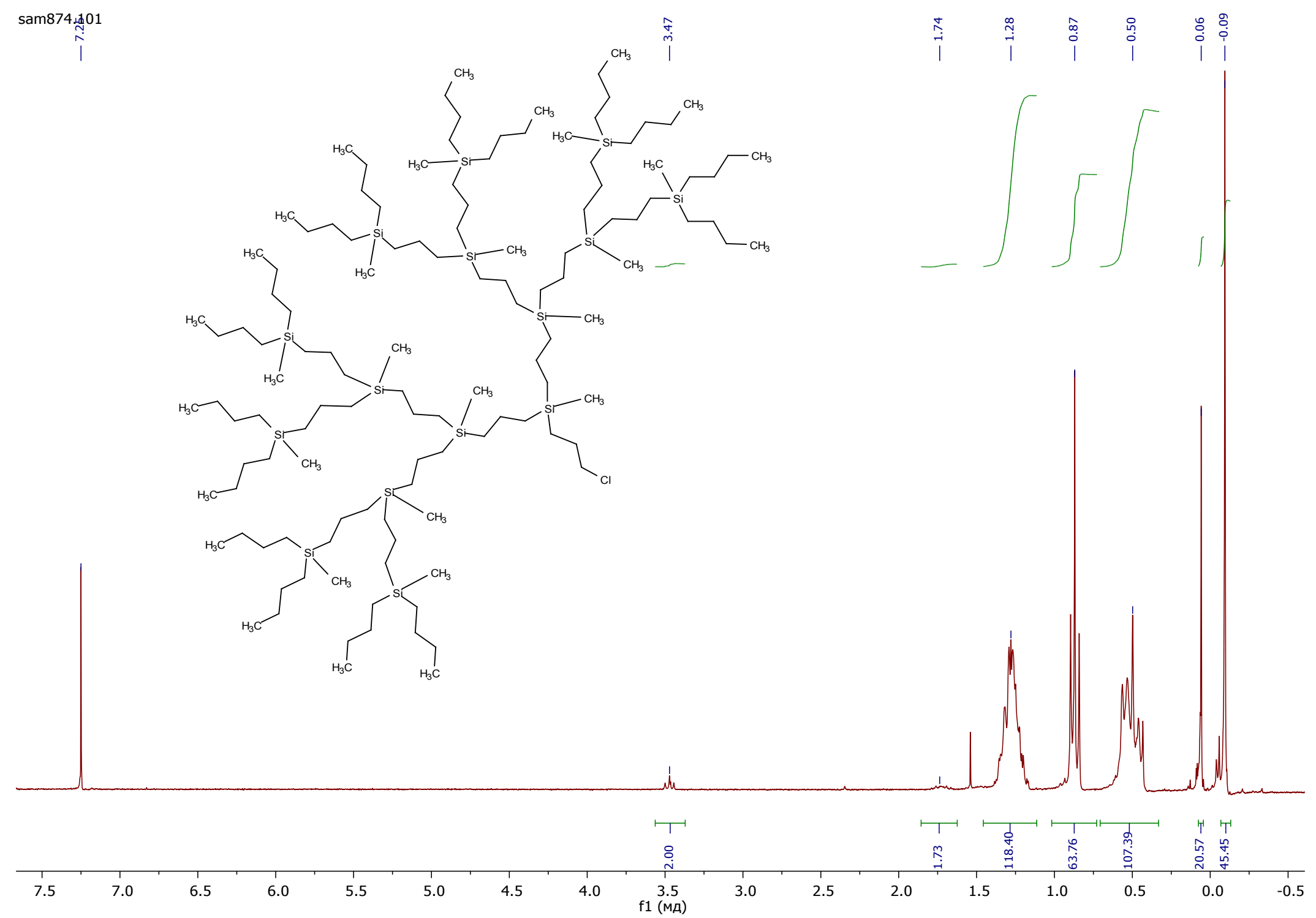


${ }^{13} \mathrm{C}$ NMR (75.5 MHz, $\mathrm{CDCl}_{3}$ of compound 13

sam874.111.3.fid

$13 \mathrm{C}$

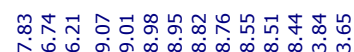

i⿱宀

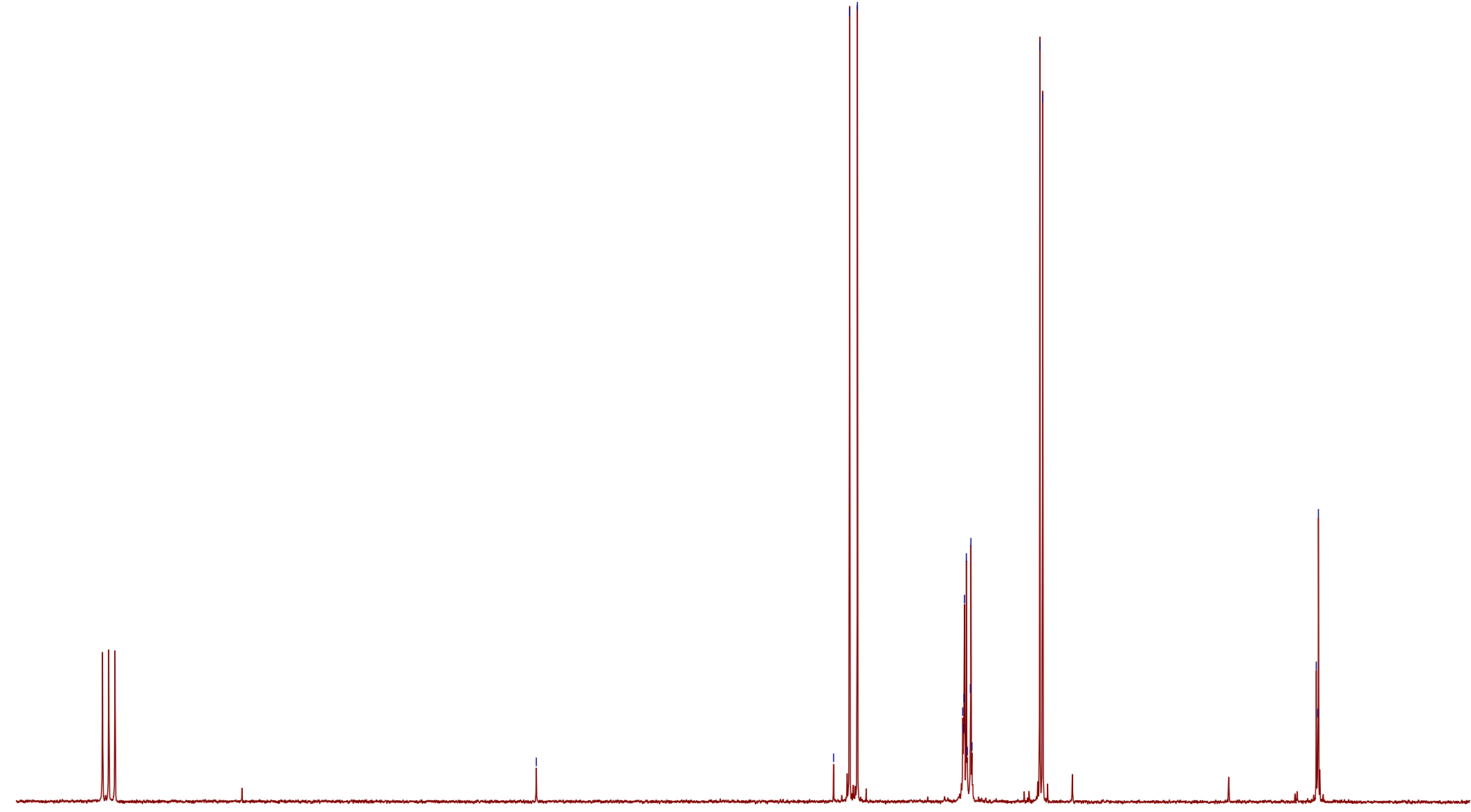


${ }^{29} \mathrm{Si} \mathrm{NMR}\left(59.6 \mathrm{MHz}, \mathrm{CDCl}_{3}\right)$ of compound 13

sam874.111.2903.fid 29Si_PO=pi $/ 8 \mathrm{~d} 1=3 \mathrm{~J}=20$

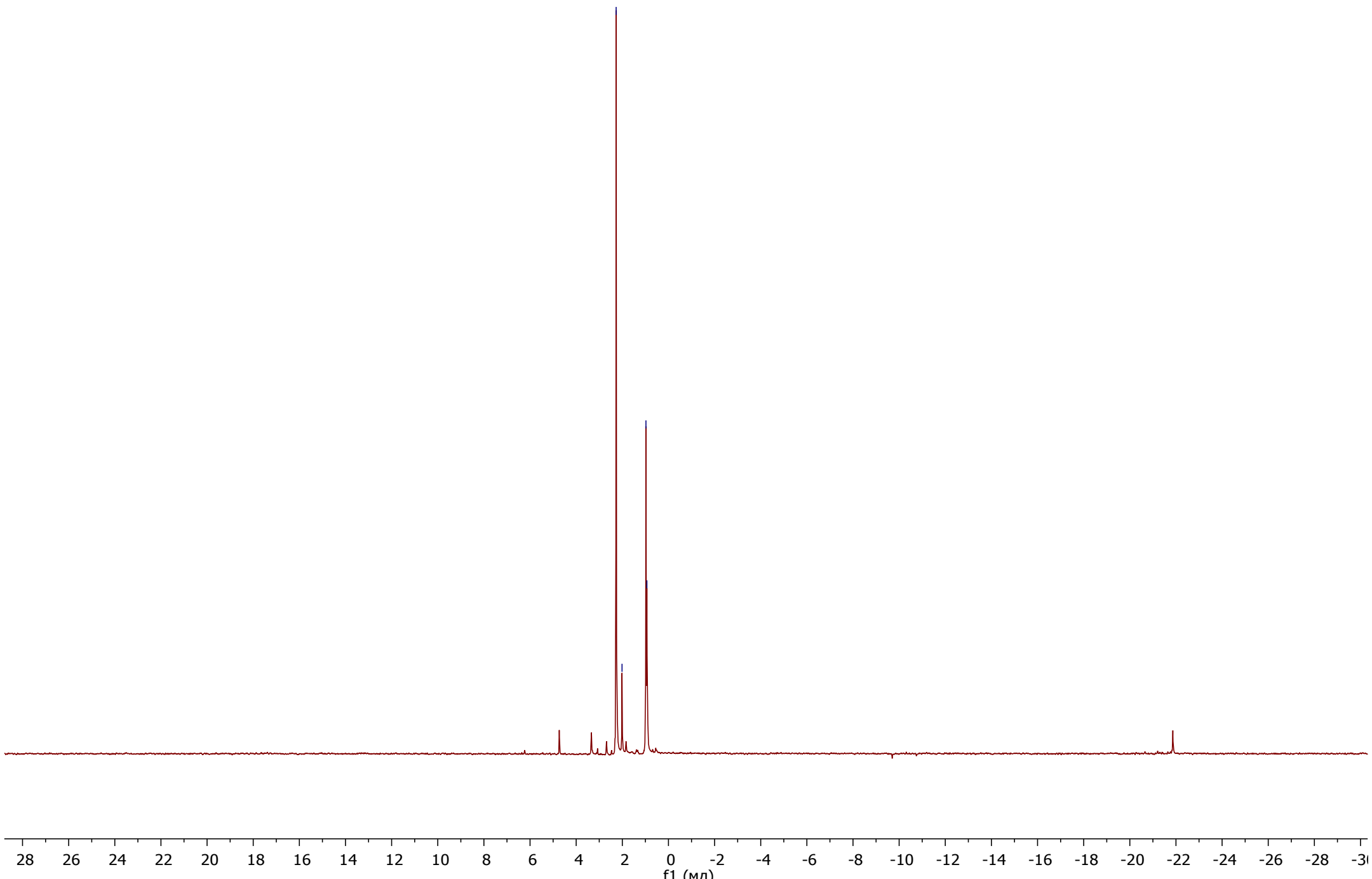


GPC curve of compound 13

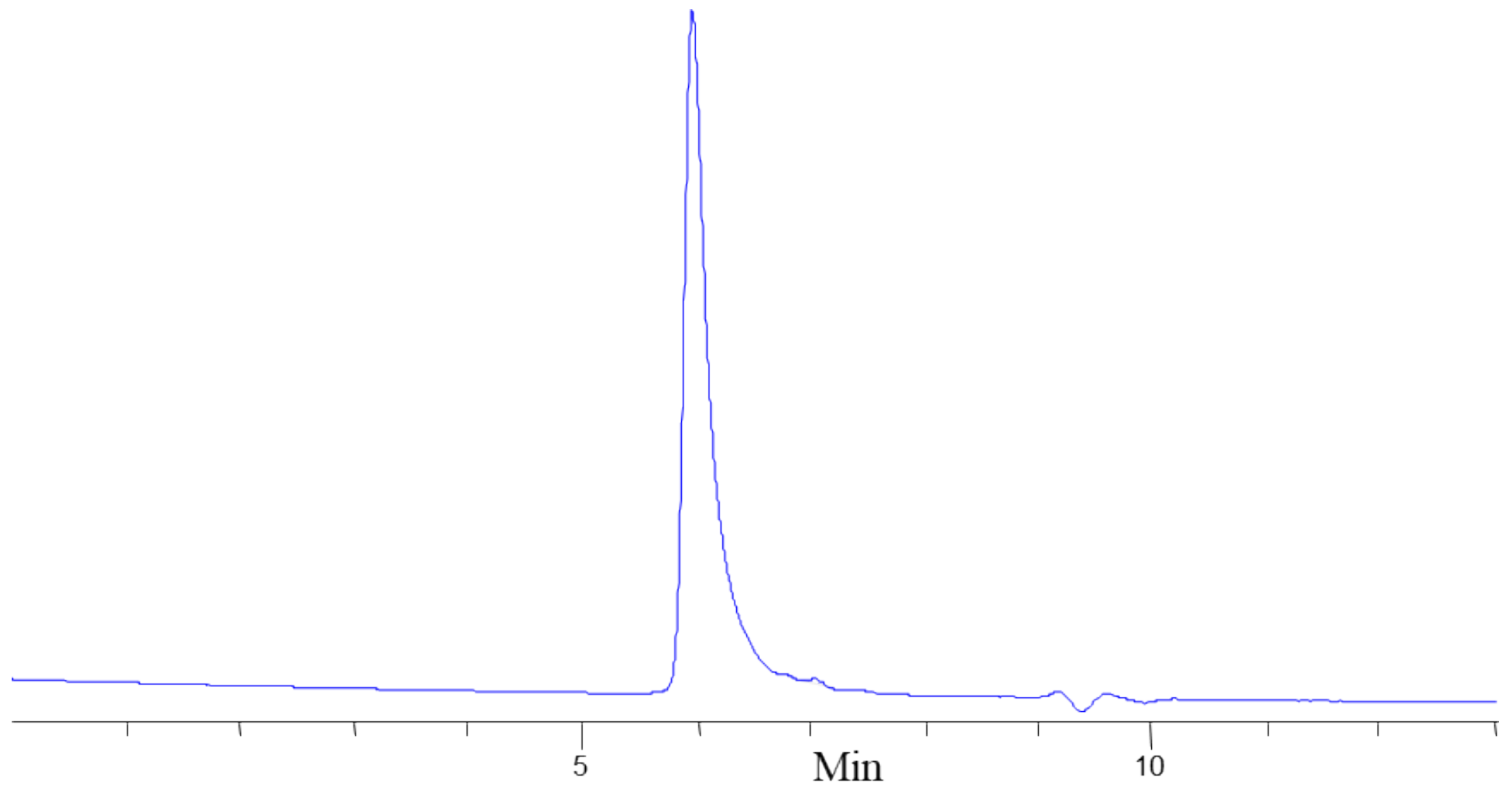


${ }^{1} \mathrm{H}$ NMR (300 MHz, $\mathrm{CDCl}_{3}$ ) of compound 10

sam913.111_D2Si7Bu8(N3).1.fid $1 \mathrm{H}$

$\stackrel{\infty}{\infty}$

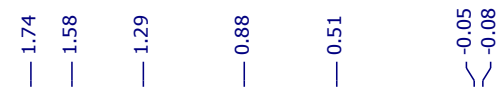

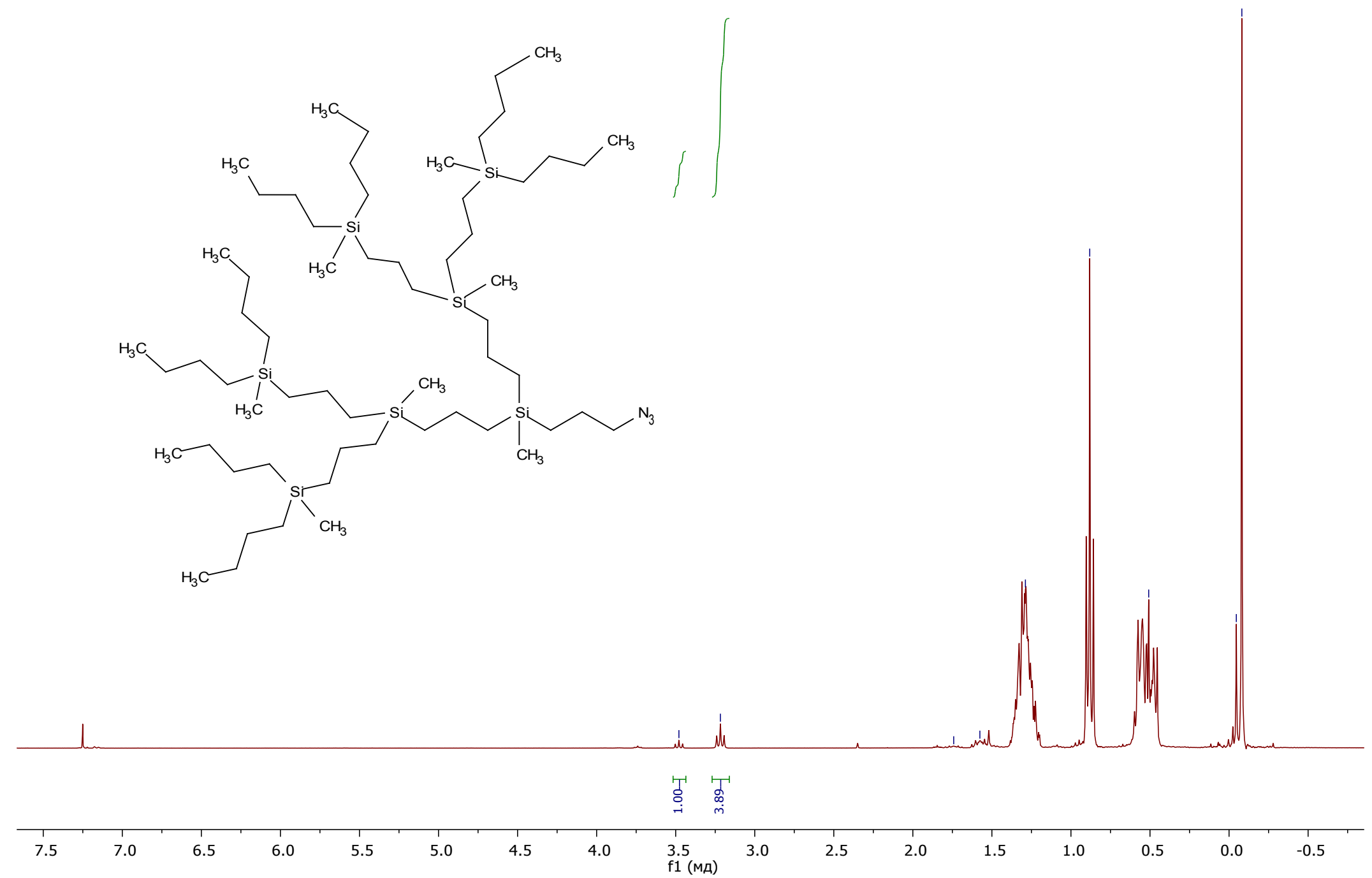


${ }^{13} \mathrm{C}$ NMR (75.5 MHz, $\mathrm{CDCl}_{3}$ of compound 10

sam913.111_D2Si7Bu8(N3).3.fid $13 \mathrm{C}$

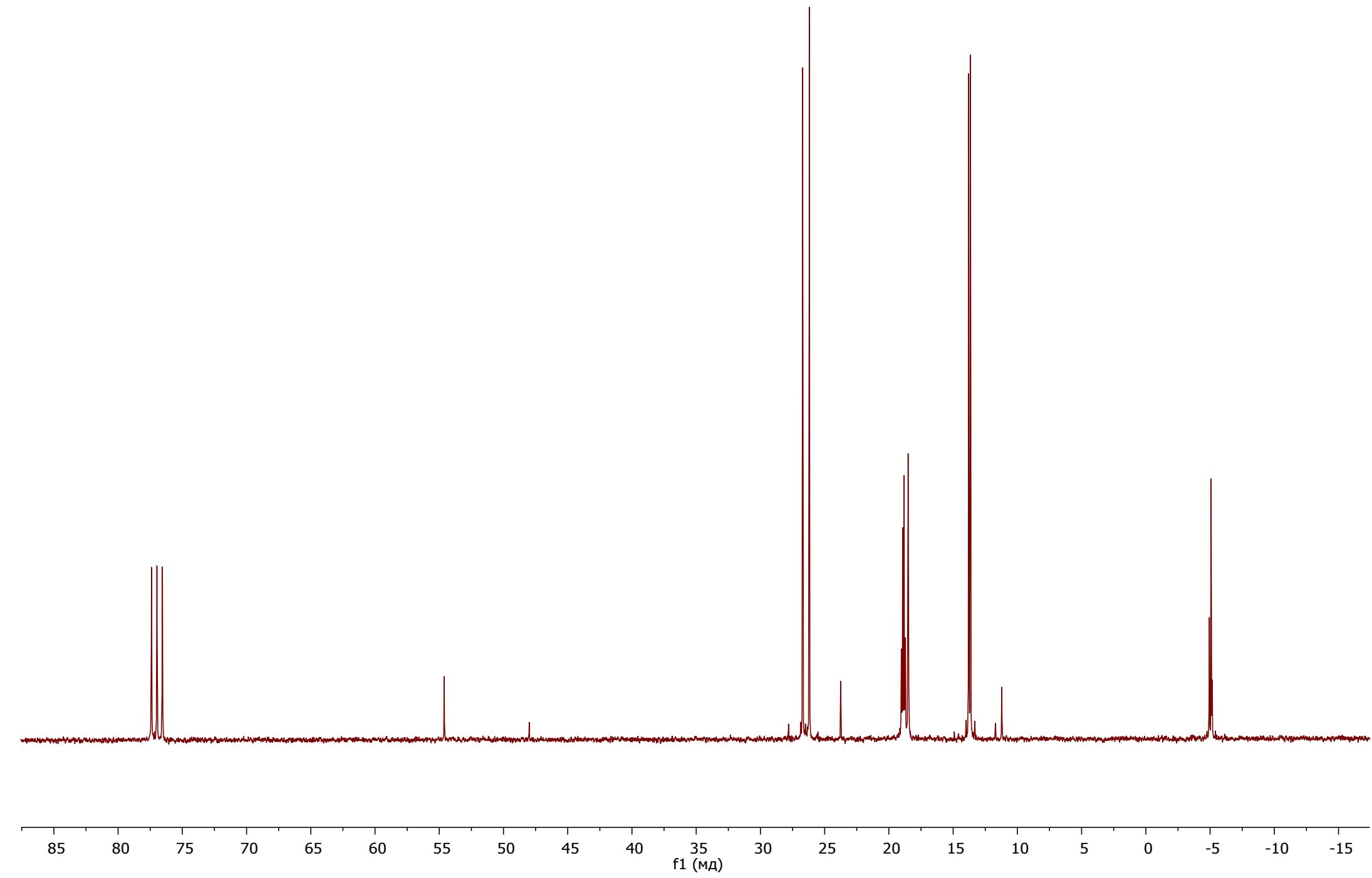


${ }^{29} \mathrm{Si} \mathrm{NMR}\left(59.6 \mathrm{MHz}, \mathrm{CDCl}_{3}\right)$ of compound 10

sam913.111_D2Si7Bu8(N3).2903.fid 29Si $\_$P0 $=$pi $/ 8 \mathrm{~d} 1=3 \mathrm{~J}=20$

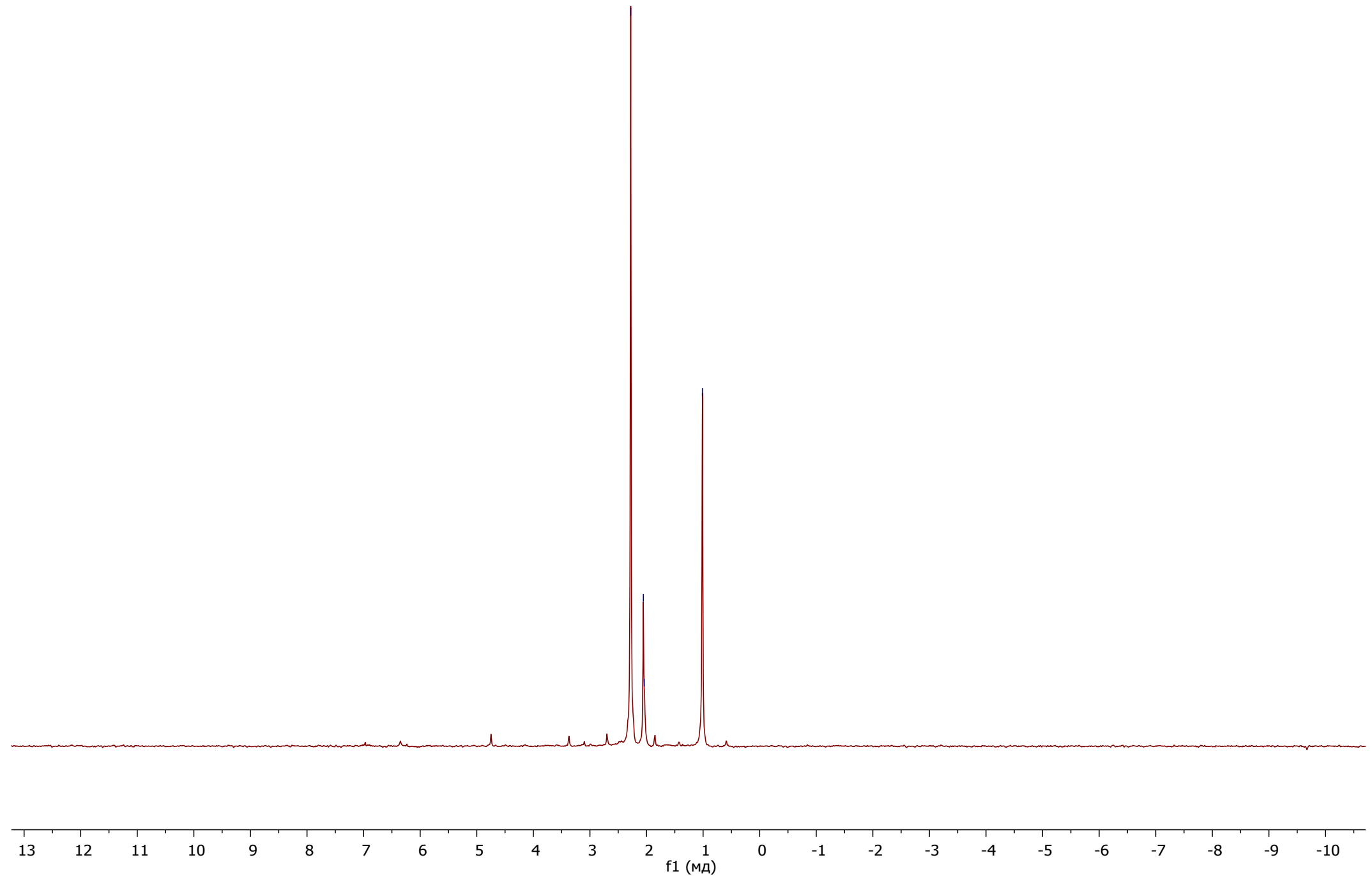


GPC curve of compound 10

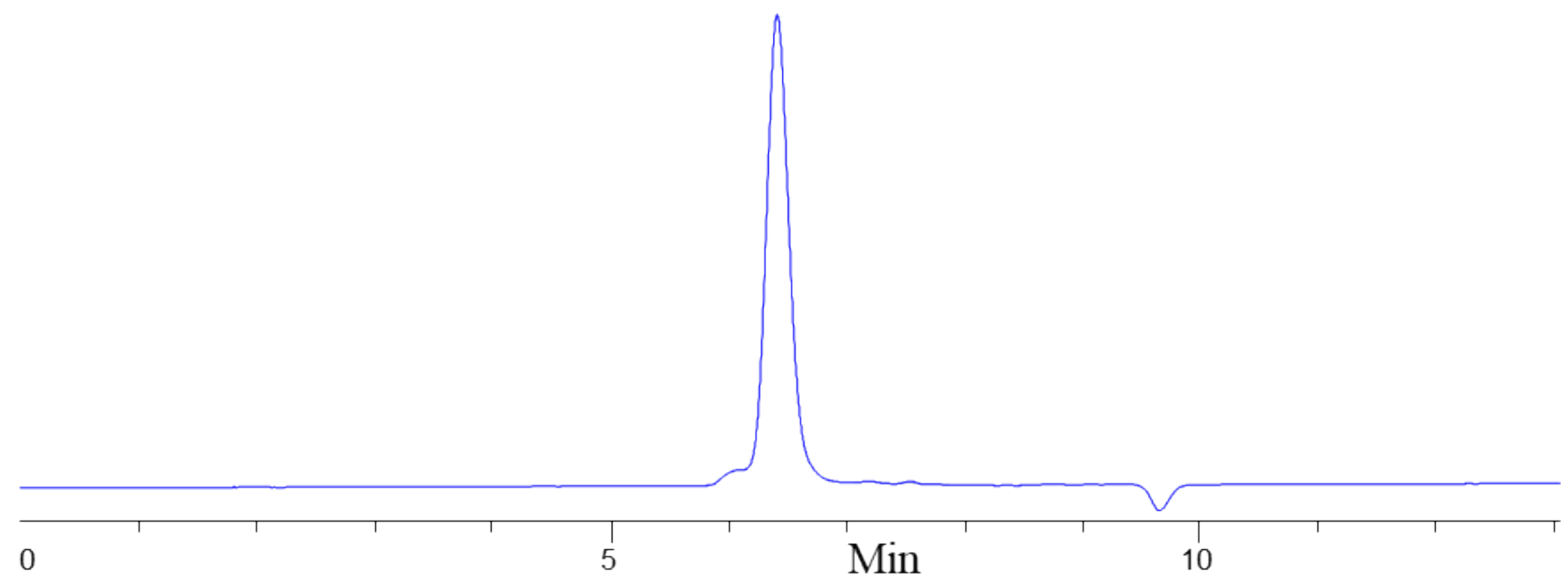


${ }^{1} \mathrm{H}$ NMR (300 MHz, $\mathrm{CDCl}_{3}$ ) of compound 14

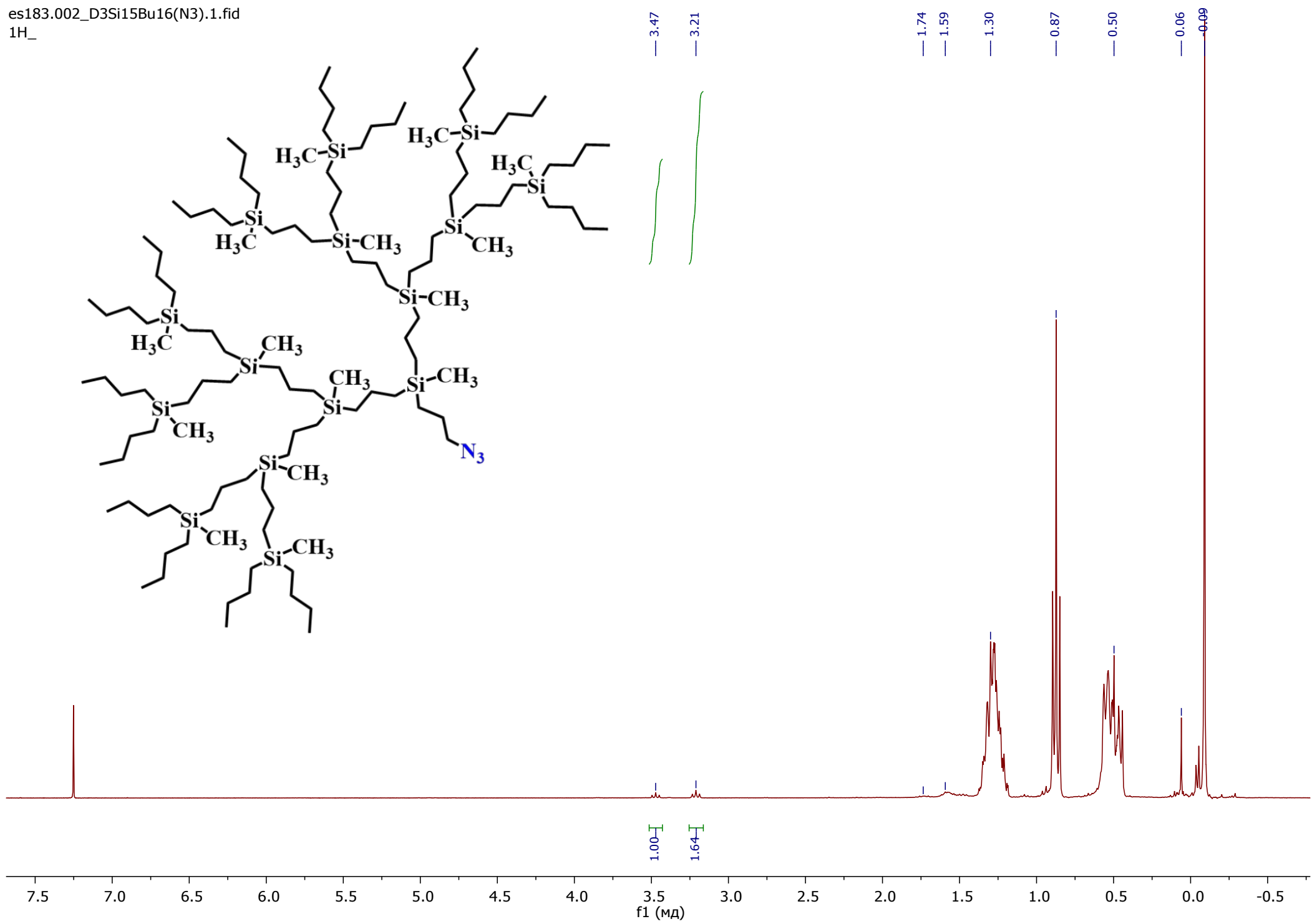


GPC curve of compound 14

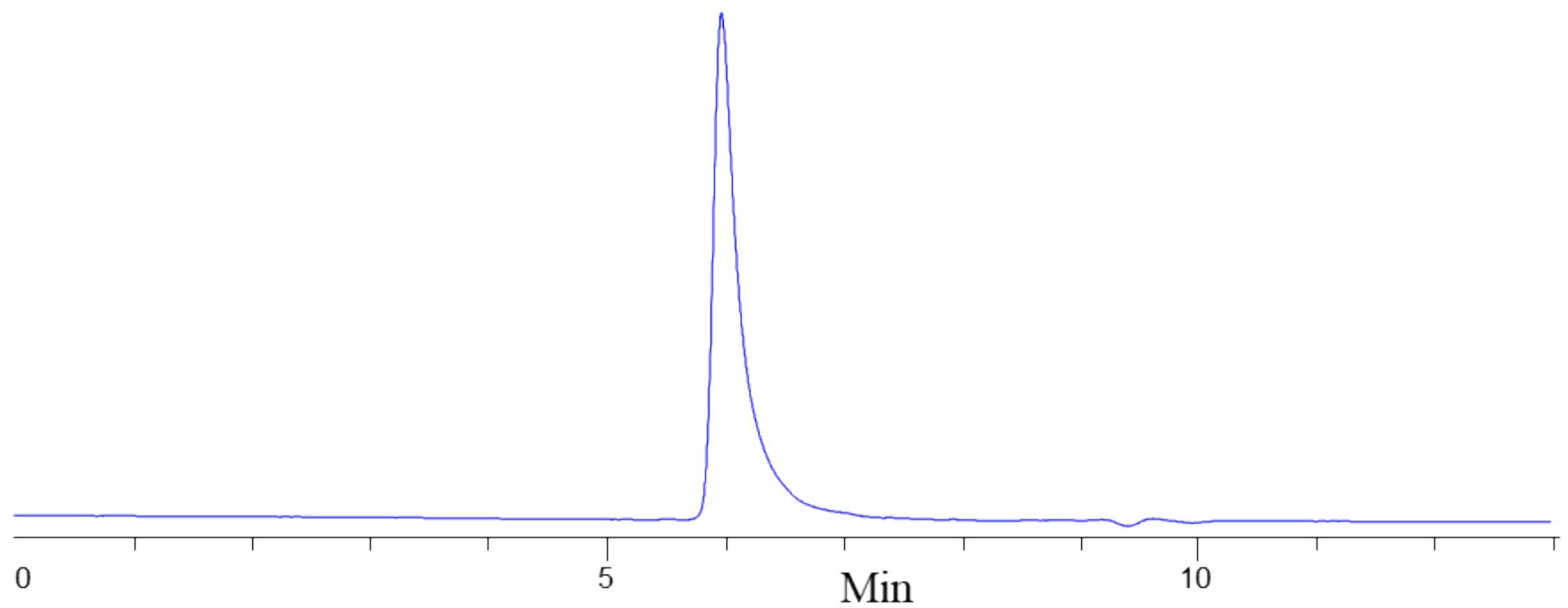


${ }^{1} \mathrm{H}$ NMR $\left(300 \mathrm{MHz}, \mathrm{CDCl}_{3}\right)$ of compound $\mathrm{G} 2$

Sample: $62 \mathrm{Ph}_{16} \mathrm{Si}_{56} \mathrm{Bu}_{64} \mid 1 \mathrm{H}-\mathrm{NMR}(300 \mathrm{MHz})$ | Solvent: $\mathrm{CDCl} 3, \mathrm{~T}=343 \mathrm{~K}$
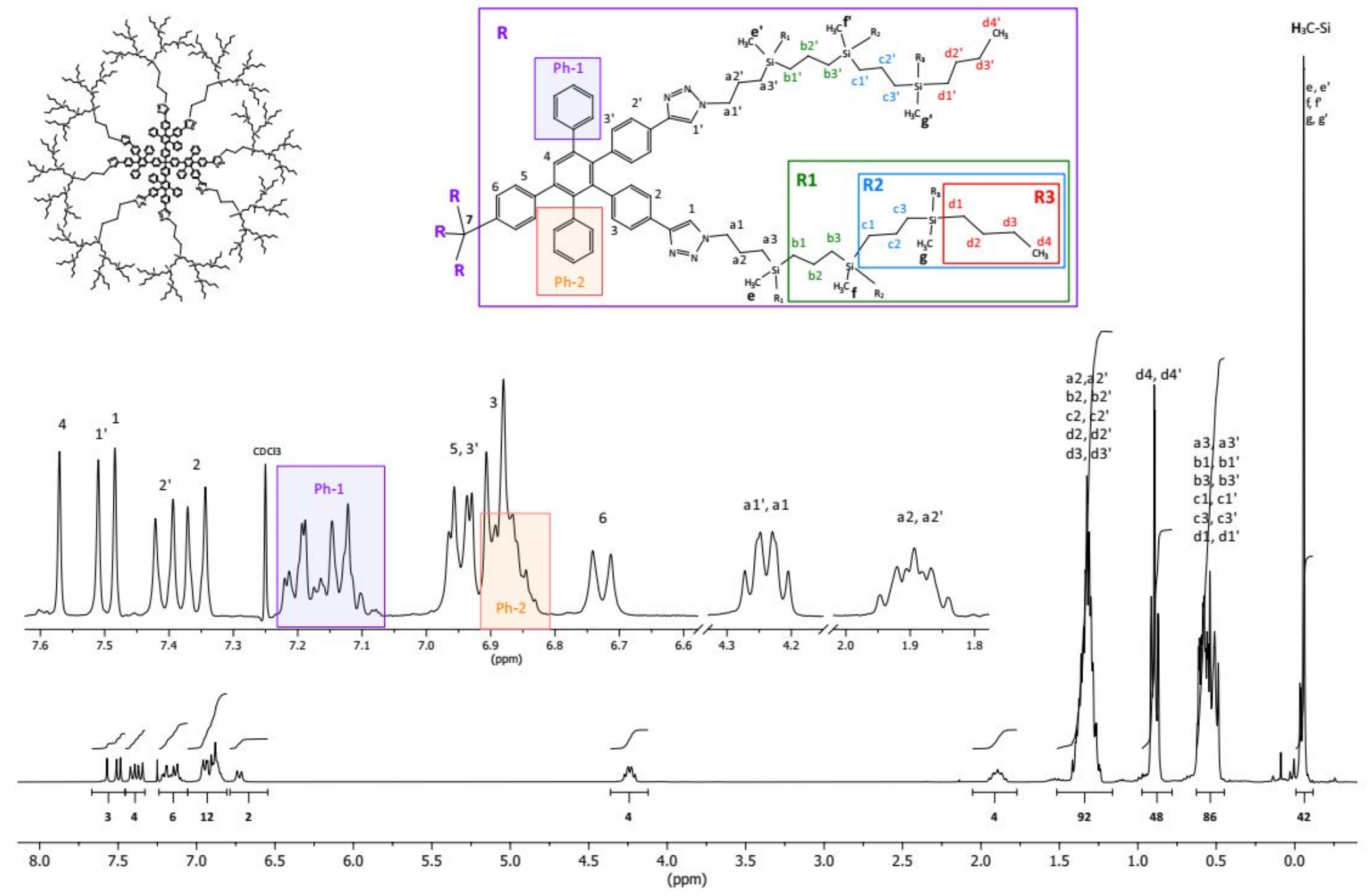
${ }^{13} \mathrm{C}$ NMR (75.5 MHz, $\mathrm{CDCl}_{3}$ of compound G2

Sample: $\mathrm{G}_{2} \mathrm{Ph}_{16} \mathrm{Si}_{56} \mathrm{Bu}_{64} \mid$ | 13C-NMR (75.5 MHz) | Solvent: CDCl3, T= 343K

${ }^{13} \mathrm{C} \mathrm{NMR}\left(75 \mathrm{MHz}^{\mathrm{C}} \mathrm{CDCl}_{3}\right) \delta$ 147.73, 144.78, 141.95, 141.29,

$141.17,141.09,140.39,140.14,140.09,139.82,139.58,139.07$,

$132.13,132.07,131.76,131.26,130.55,130.08,128.79,128.40$,

$128.19,127.71,126.96,126.36,125.74,124.59,124.36,119.09$,

$63.88,53.47,26.63,26.31,25.43,25.41,19.29,19.17,19.09$,

$18.86,18.65,18.59,13.90,13.67,11.60,-4.83,-4.93,-5.11$
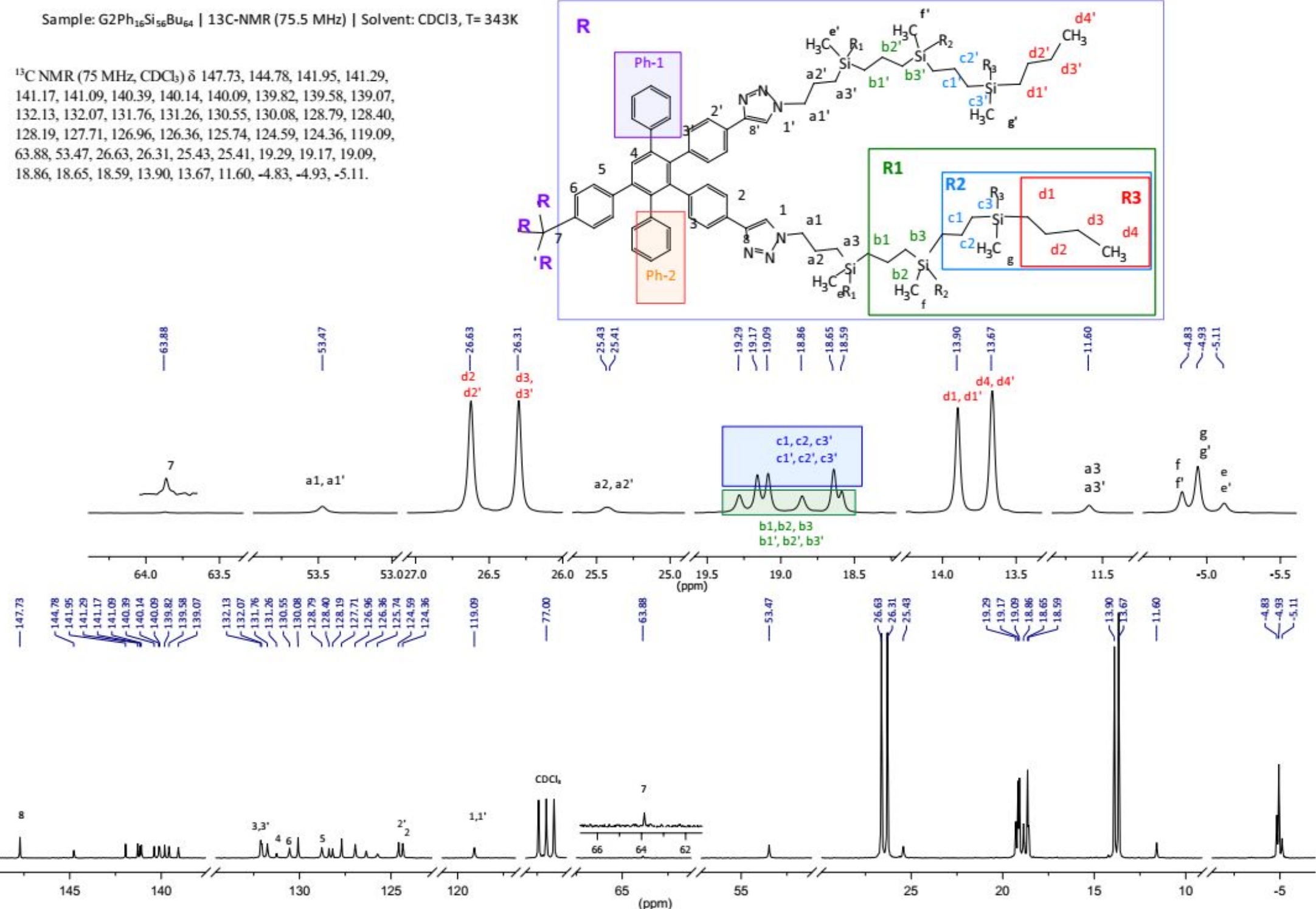
Sample: G2, A fragment of HSQC ${ }^{1} \mathrm{H}-{ }^{13} \mathrm{C}$ NMR spectrum ( aromatic region )

Sample: $\mathrm{G}_{2} \mathrm{Ph}_{16} \mathrm{Si}_{56} \mathrm{Bu}_{64} \mid \mathrm{A}$ fragment of $\mathrm{HSQC}{ }^{1} \mathrm{H}-{ }^{13} \mathrm{C}$ NMR spectrum (aromatic region ).
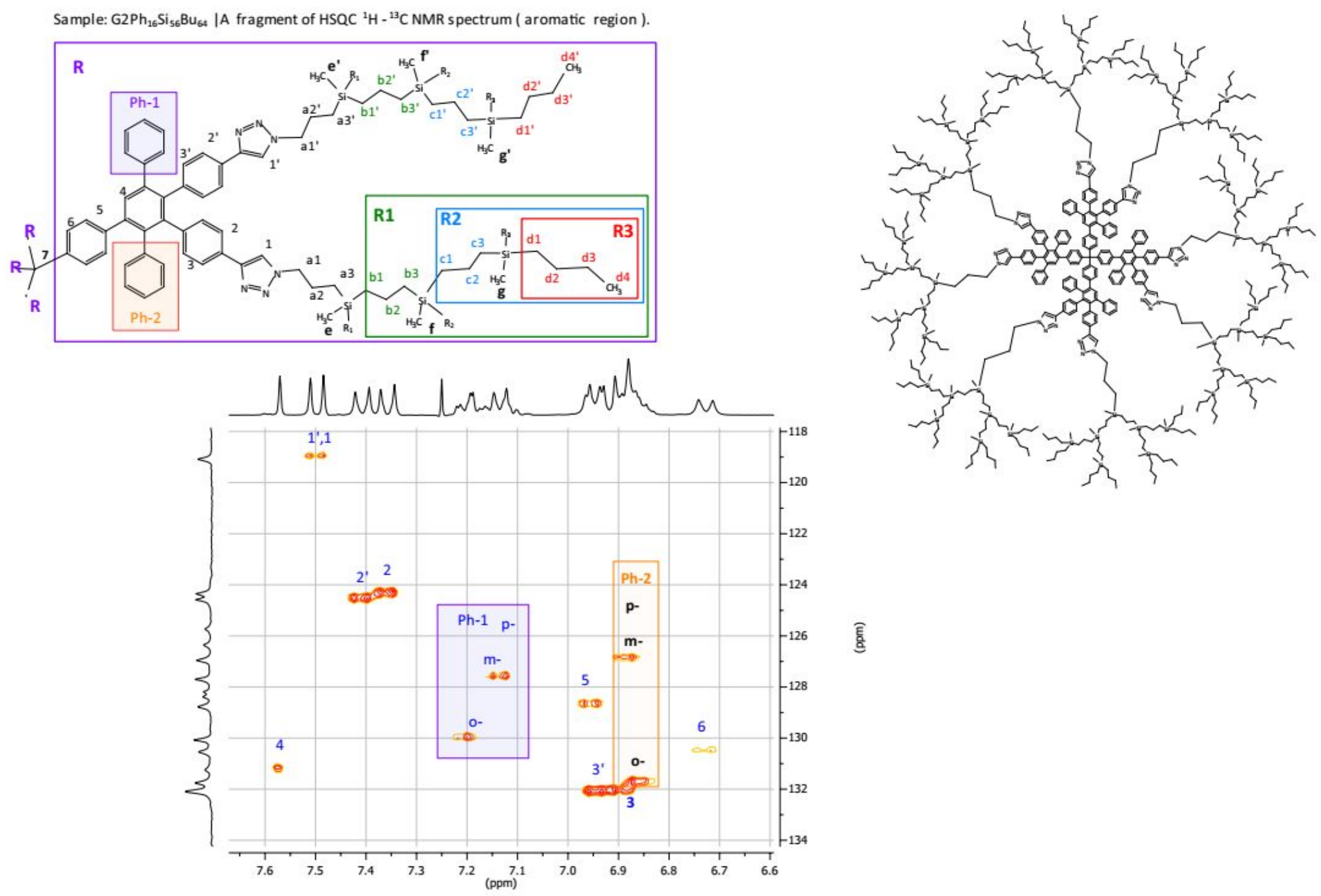

홍 
${ }^{29} \mathrm{Si} \mathrm{NMR}\left(60 \mathrm{MHz}, \mathrm{CDCl}_{3}\right)$ of compound G2

Sample: $\mathrm{G}_{2} \mathrm{Ph}_{16} \mathrm{Si}_{56} \mathrm{Bu}_{64}$ | ${ }^{29} \mathrm{Si}-\mathrm{NMR}(60 \mathrm{MHz})$ | Solvent: $\mathrm{CDCl} 3, \mathrm{~T}=343 \mathrm{~K}$

$\left.{ }^{29} \mathrm{Si} \mathrm{NMR}\left(60 \mathrm{MHz}^{\mathrm{CDCl}}\right)_{3}\right) \delta: 2.31,2.09,1.11$.

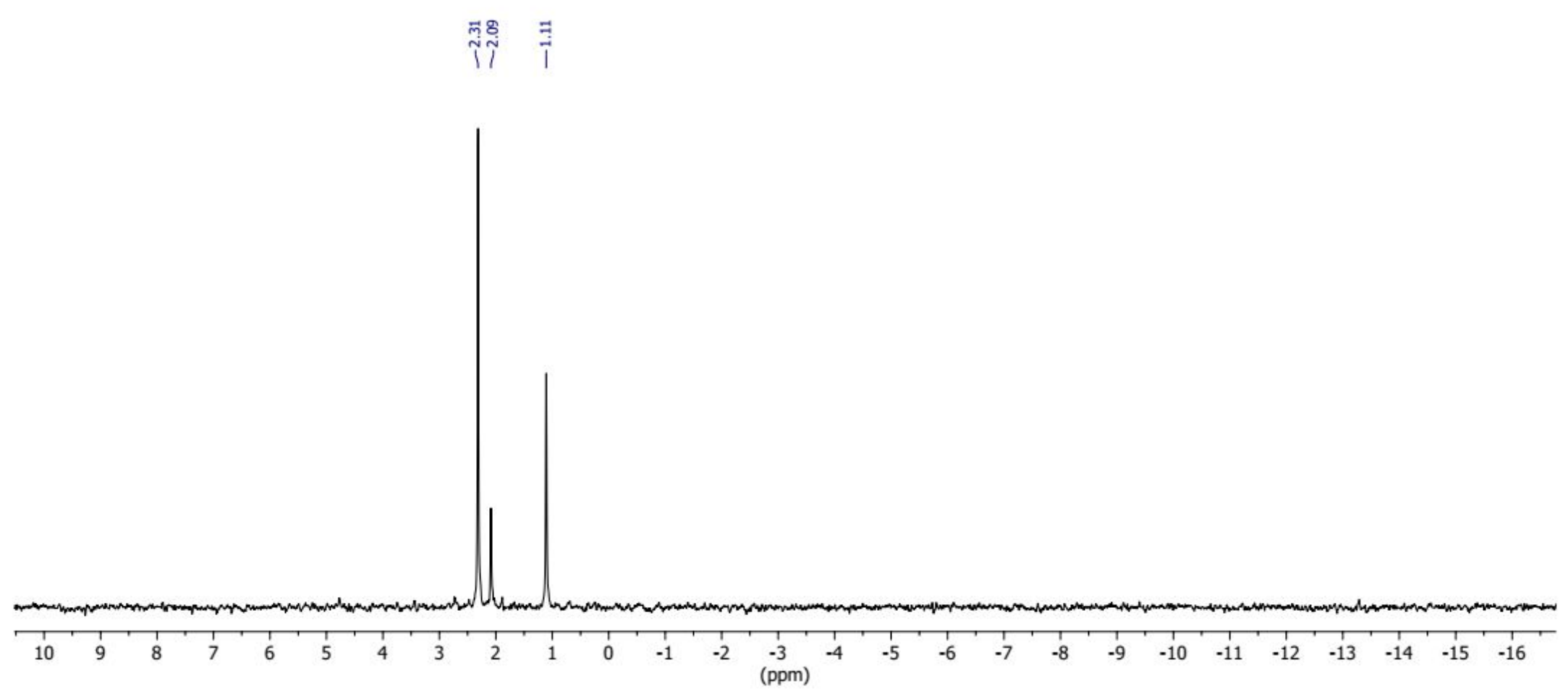


GPC curve of compound G2 before and after purification on preparative chromatography
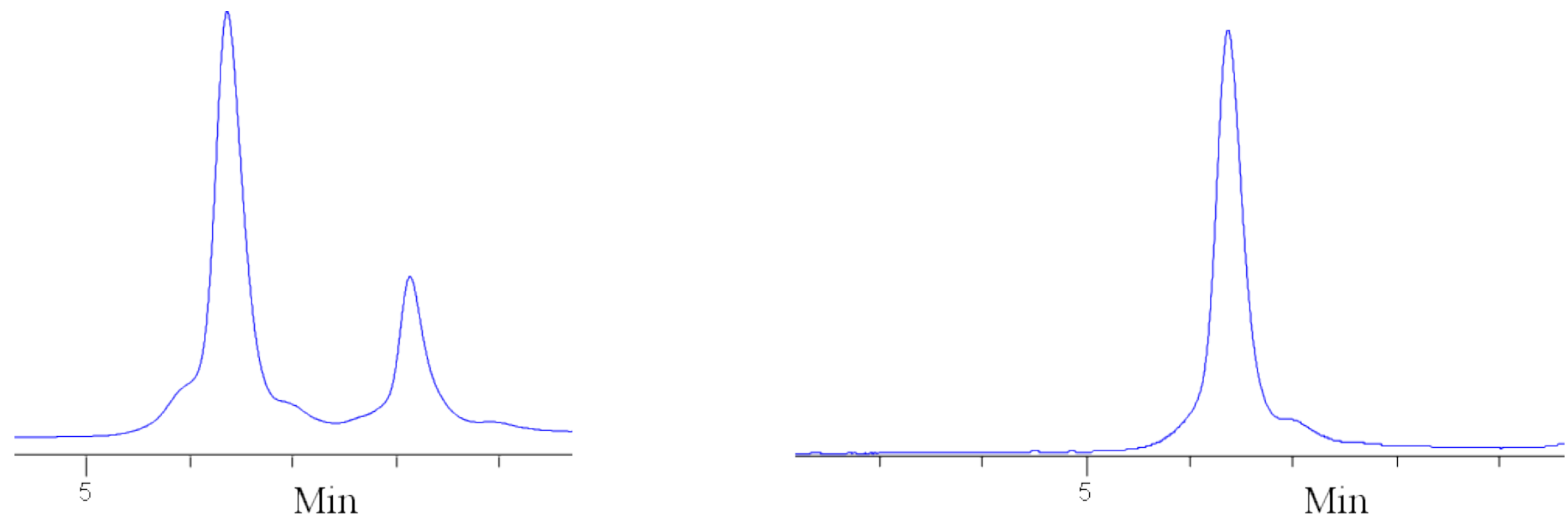
${ }^{1} \mathrm{H}$ NMR (300 MHz, $\mathrm{CDCl}_{3}$ ) of compound G3

ES218 (G3) | ${ }^{1} \mathrm{H}-\mathrm{NMR}$ (300MHz) | Solvent: $\mathrm{CDCl} 3, \mathrm{~T}=343 \mathrm{~K}$
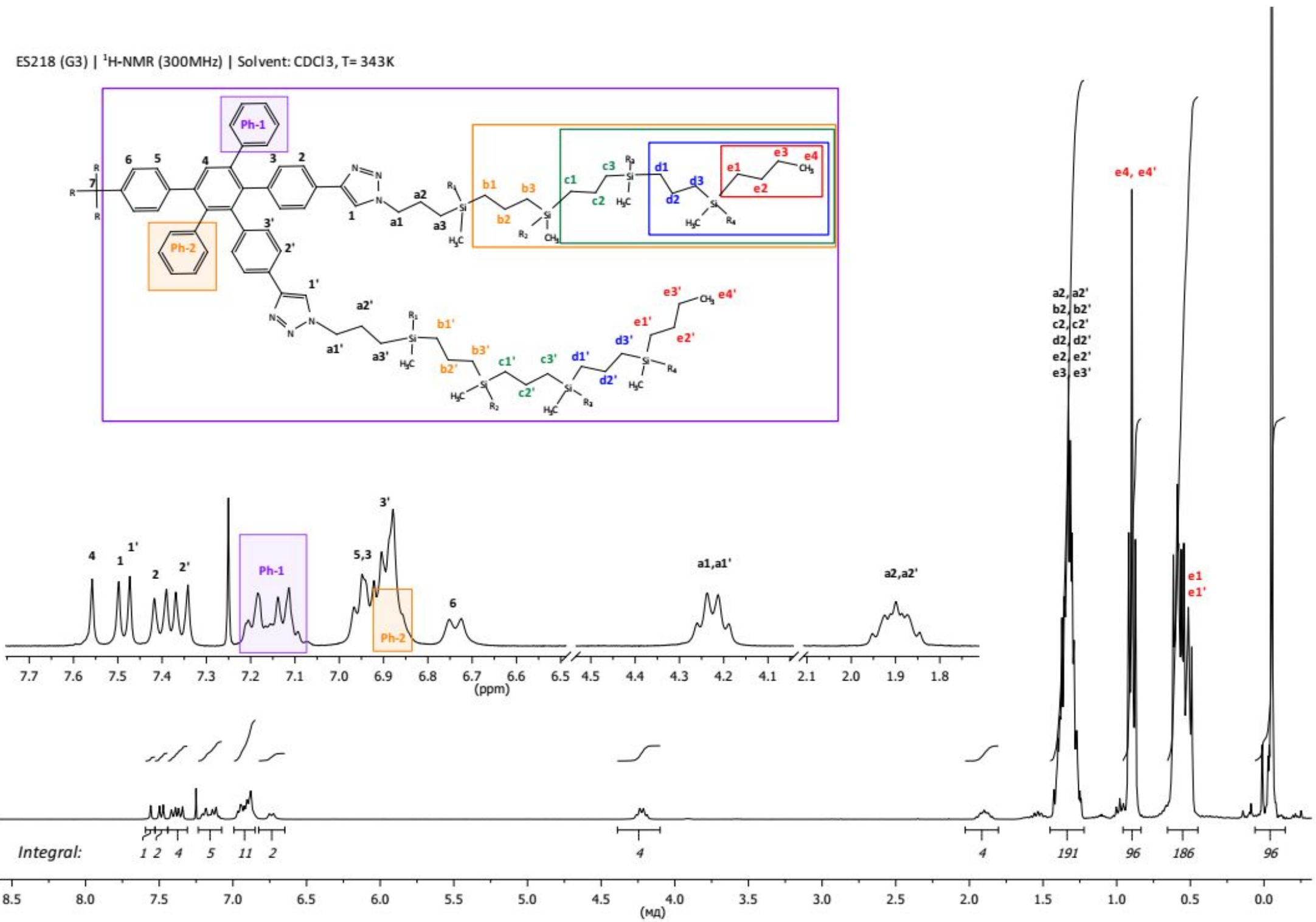
${ }^{13} \mathrm{C}$ NMR (75.5 MHz, $\mathrm{CDCl}_{3}$ of compound G3

ES218 (G3) | ${ }^{13} \mathrm{C}-\mathrm{NMR}$ (75MHz) | Solvent: CDCl3, T= 343K

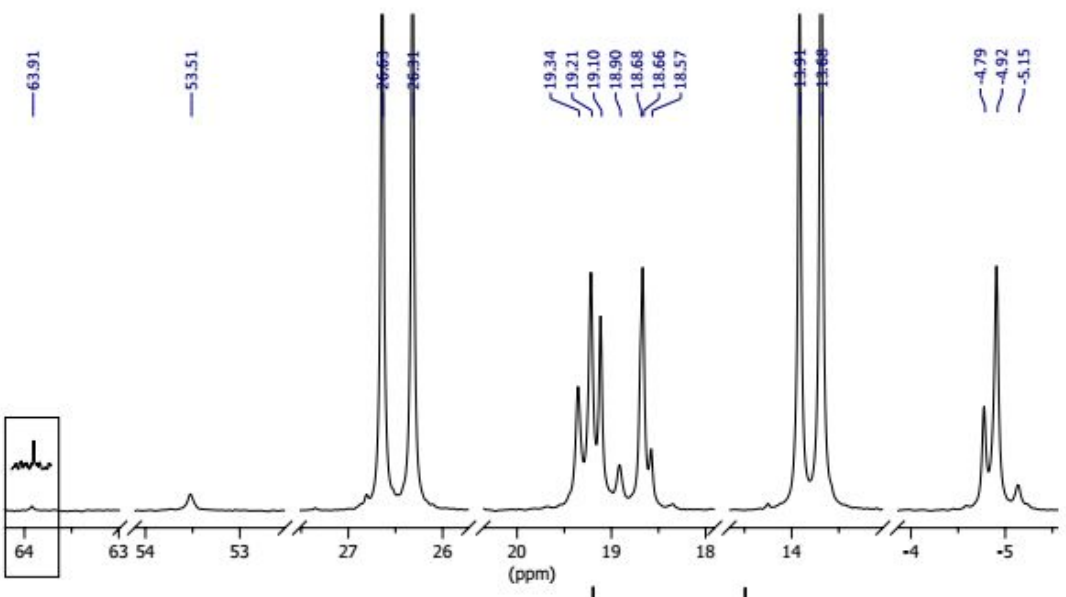

145

140

135

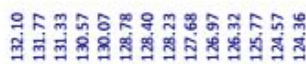

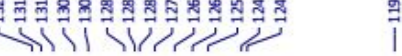

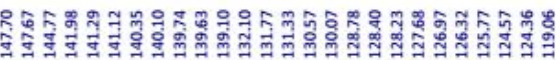

${ }^{13} \mathrm{CNMR}(75 \mathrm{MHz}, \mathrm{CDCl}) \delta 147.70,147.67,144,77,141.98,141.29,141.12,140.35,140.10$ $139.74,139.63,139.10,132.10,131.77,131.33,130.57,130.07,128.78,128.40,128.23,127.68$

$126.97,126.32,125.77,124.57,124.36,119.06,63.91,53.51,2$.
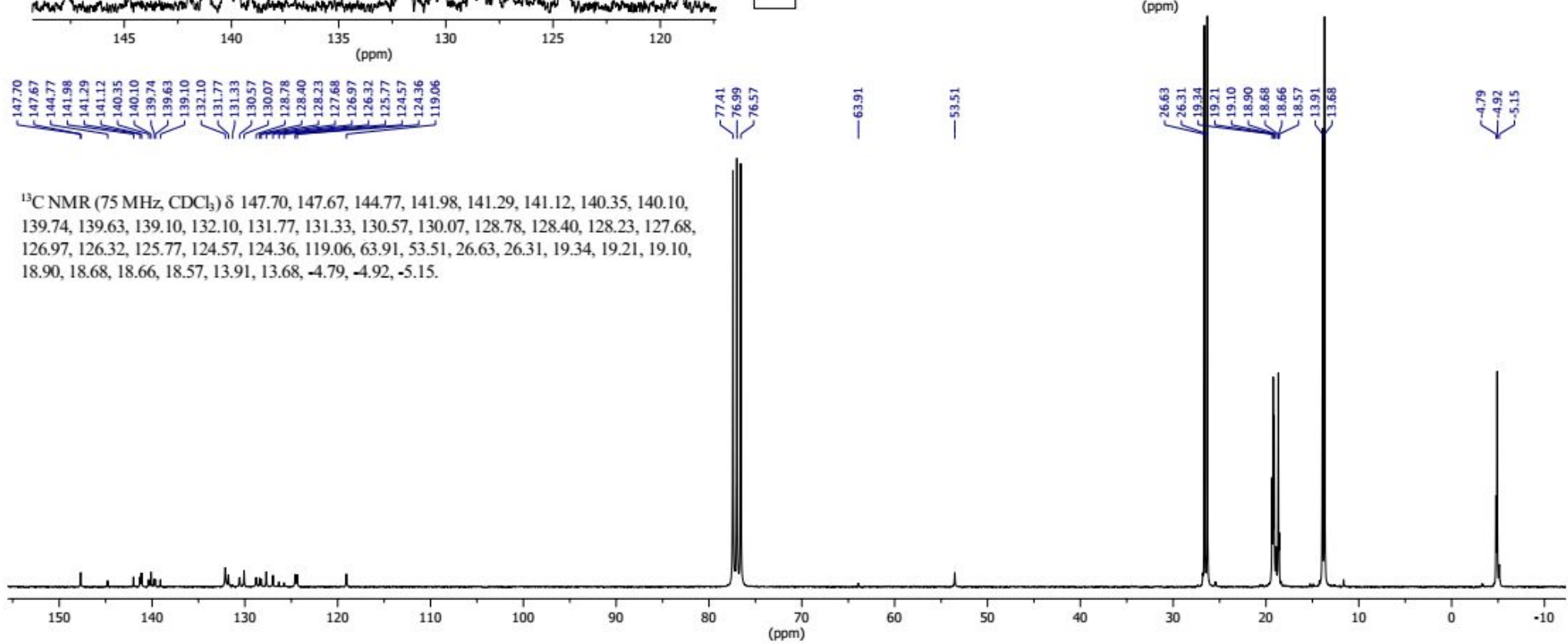
${ }^{29} \mathrm{Si}$ NMR $\left(60 \mathrm{MHz}, \mathrm{CDCl}_{3}\right)$ of compound G3

ES218 (G3) | ${ }^{29} \mathrm{Si}-\mathrm{NMR}$ (60MHz) | Solvent: CDCl3, T= 343K

${ }^{29} \mathrm{Si} \mathrm{NMR}\left(60 \mathrm{MHz}, \mathrm{CDCl}_{3}\right) \delta: 2.31,2.08,2.06,1.11,1.06$
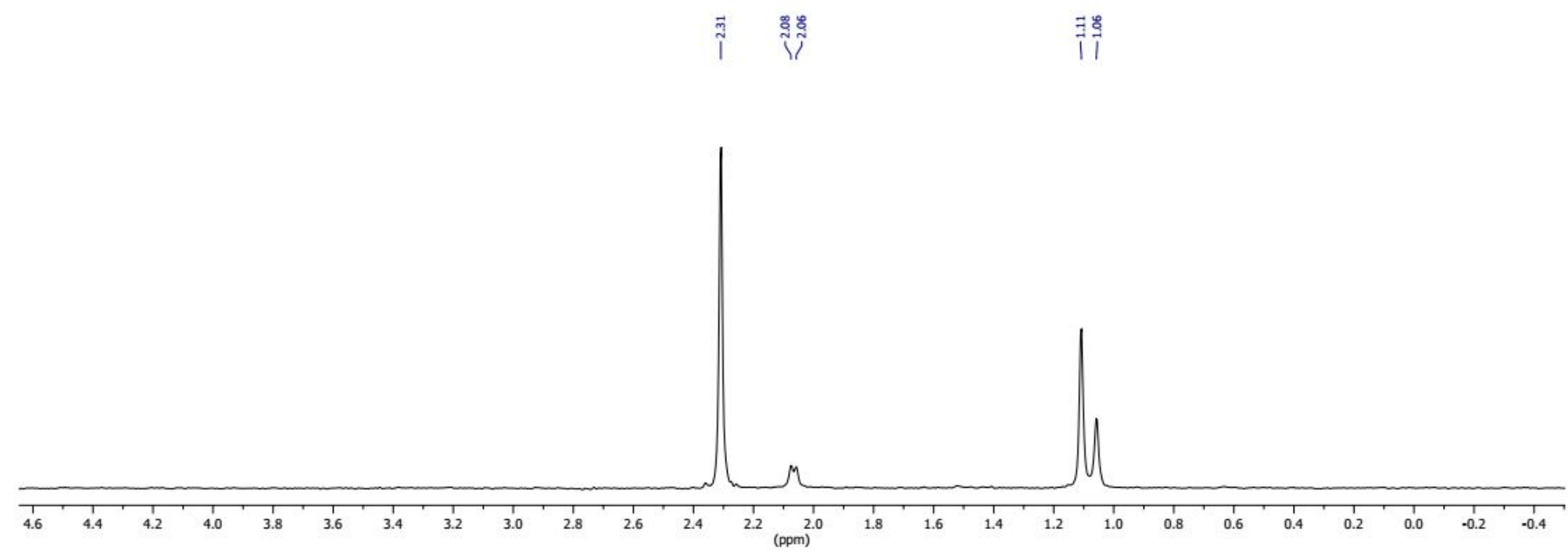
GPC curve of compound G3 before and after purification on preparative chromatography
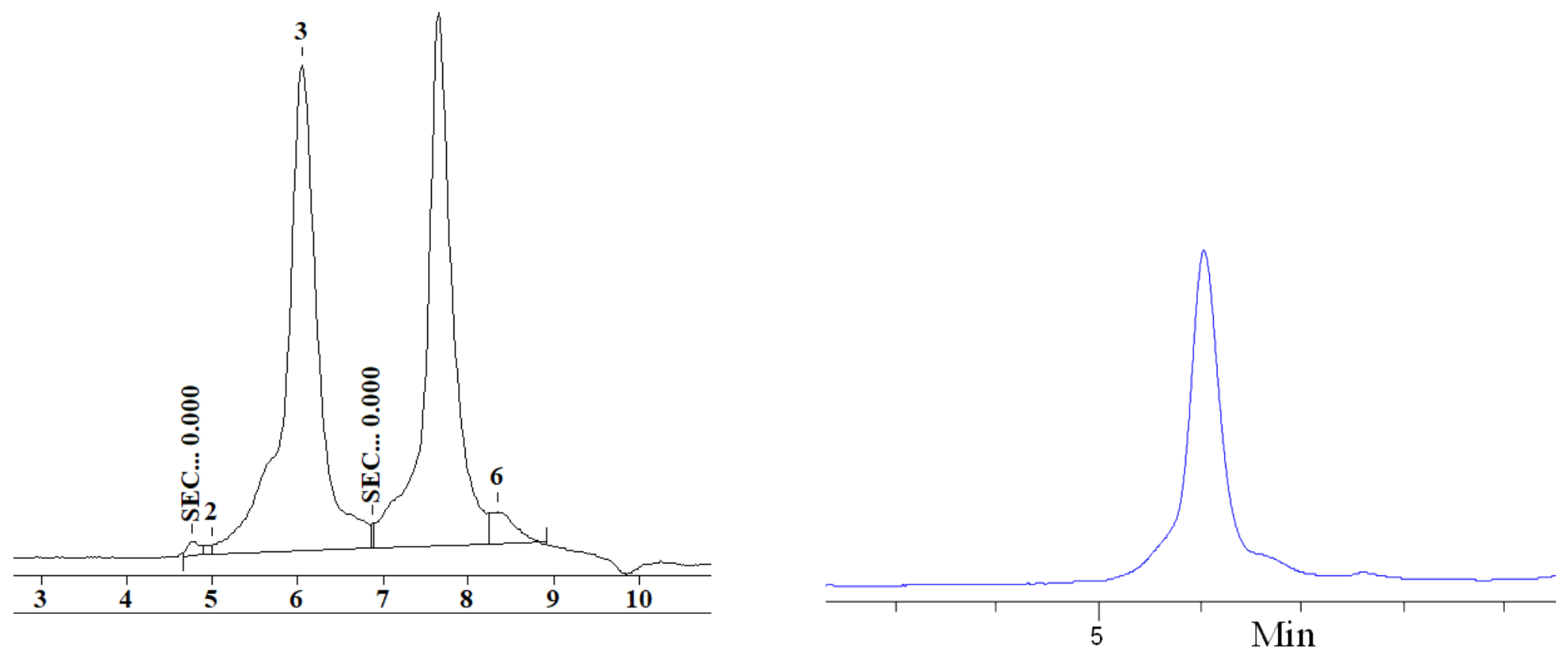
MALDI-TOF of compound G3

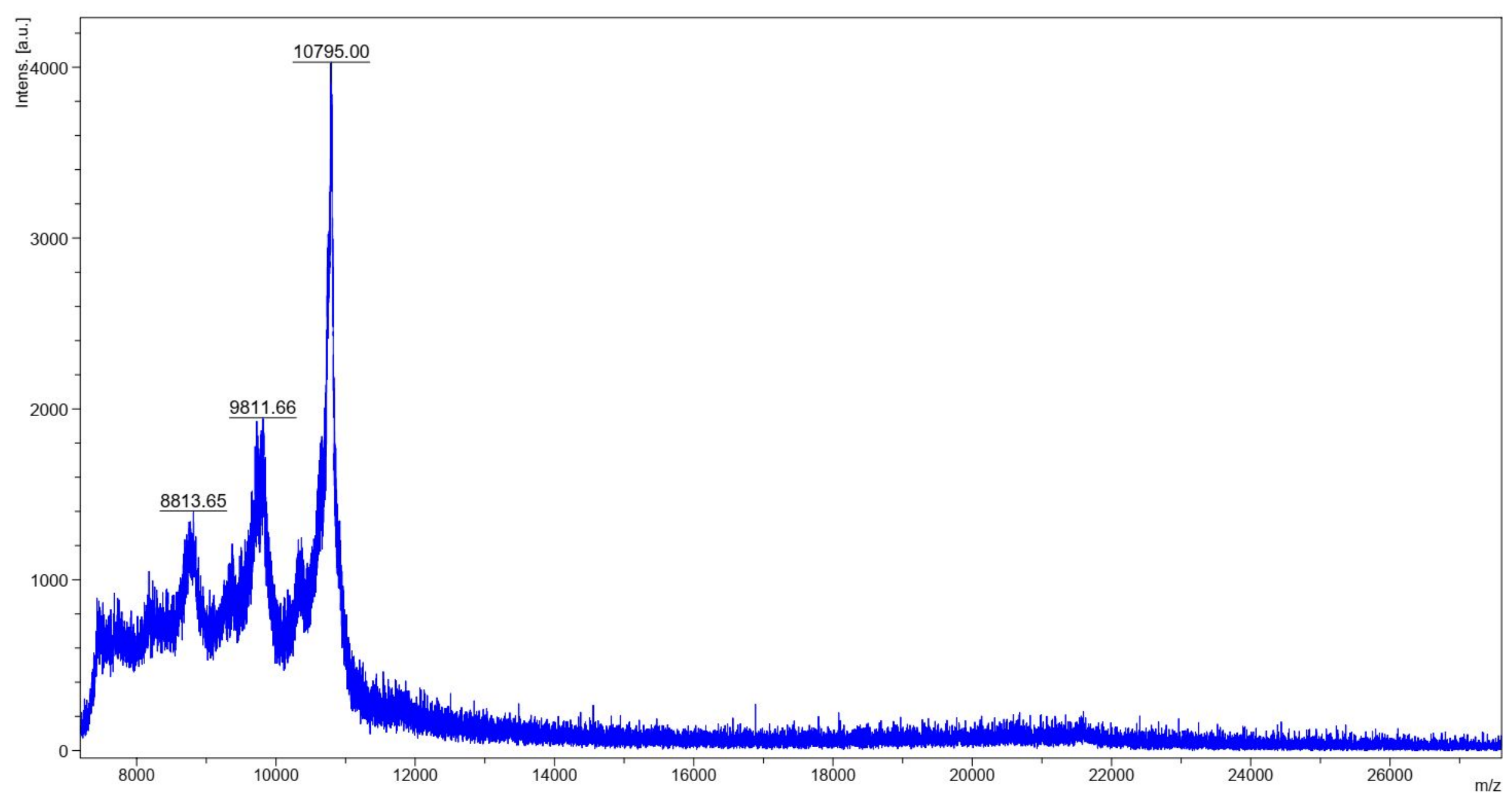


MALDI-TOF of compound G3

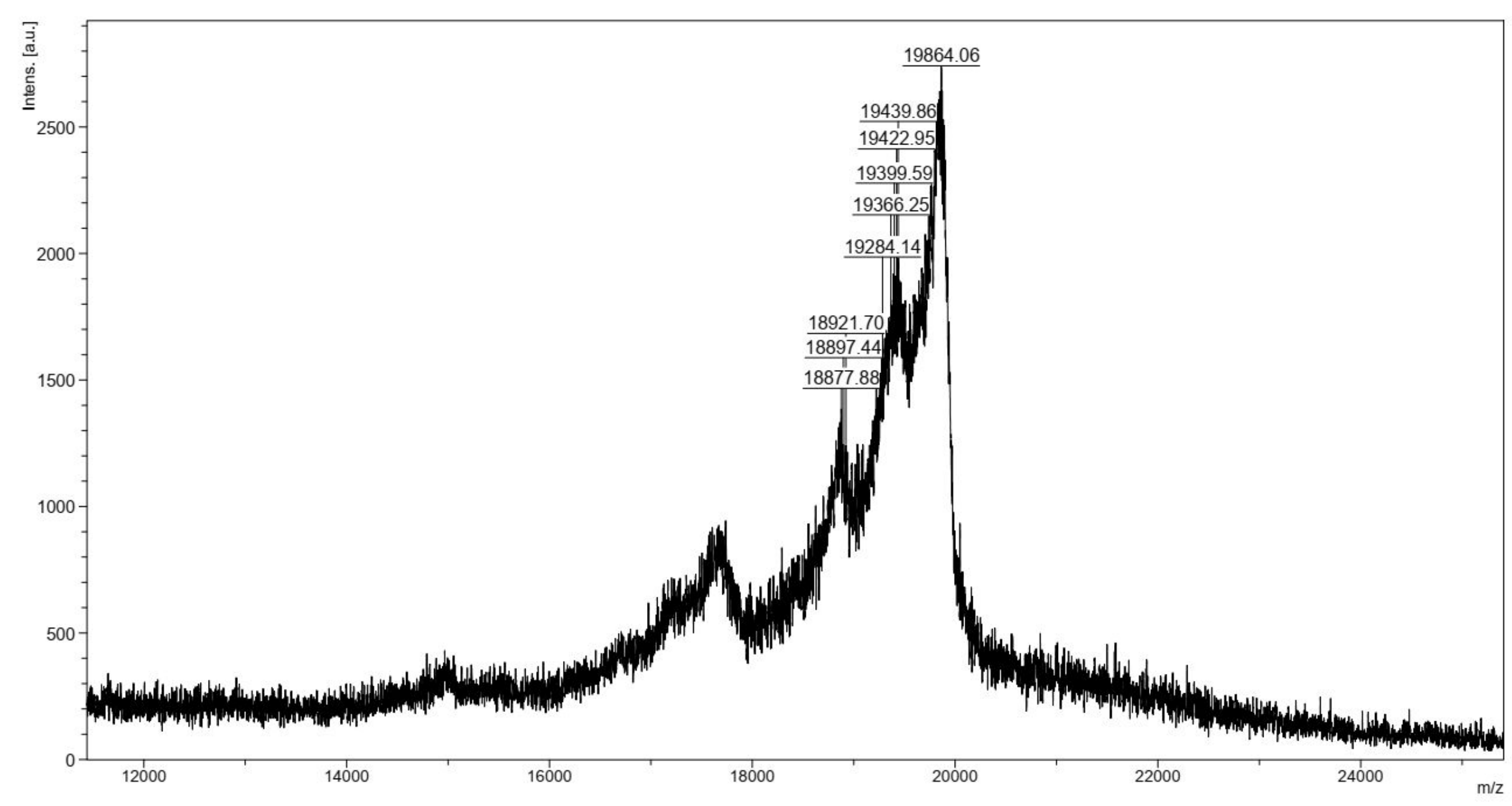




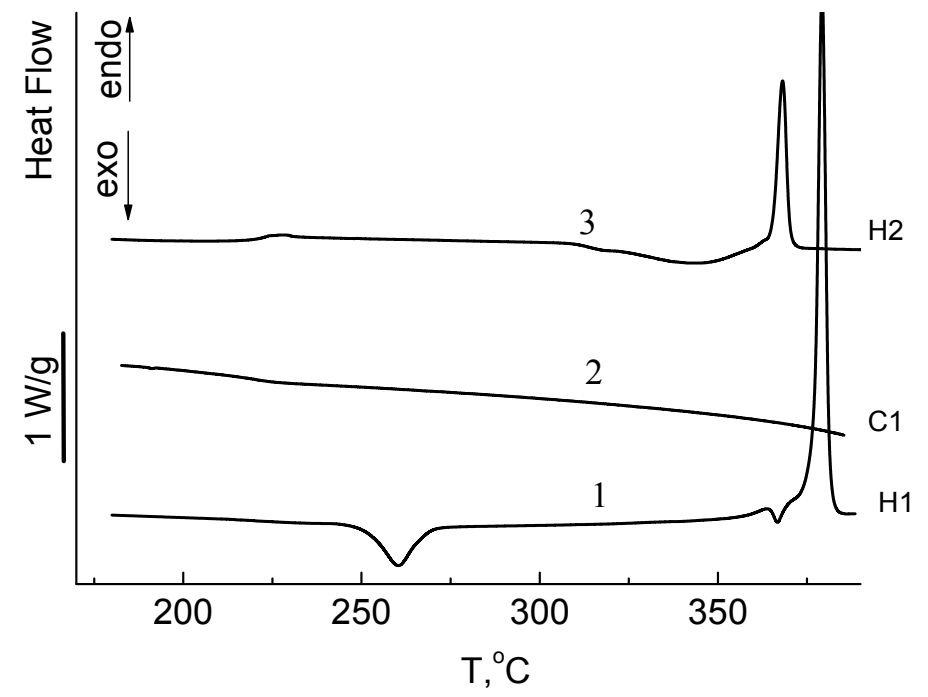

Figure S1. DSC traces of the phenylene core dendrimer. The curve 1 indicates first heating, the curve 2 is for cooling, and the curve 3 shows second heating. 


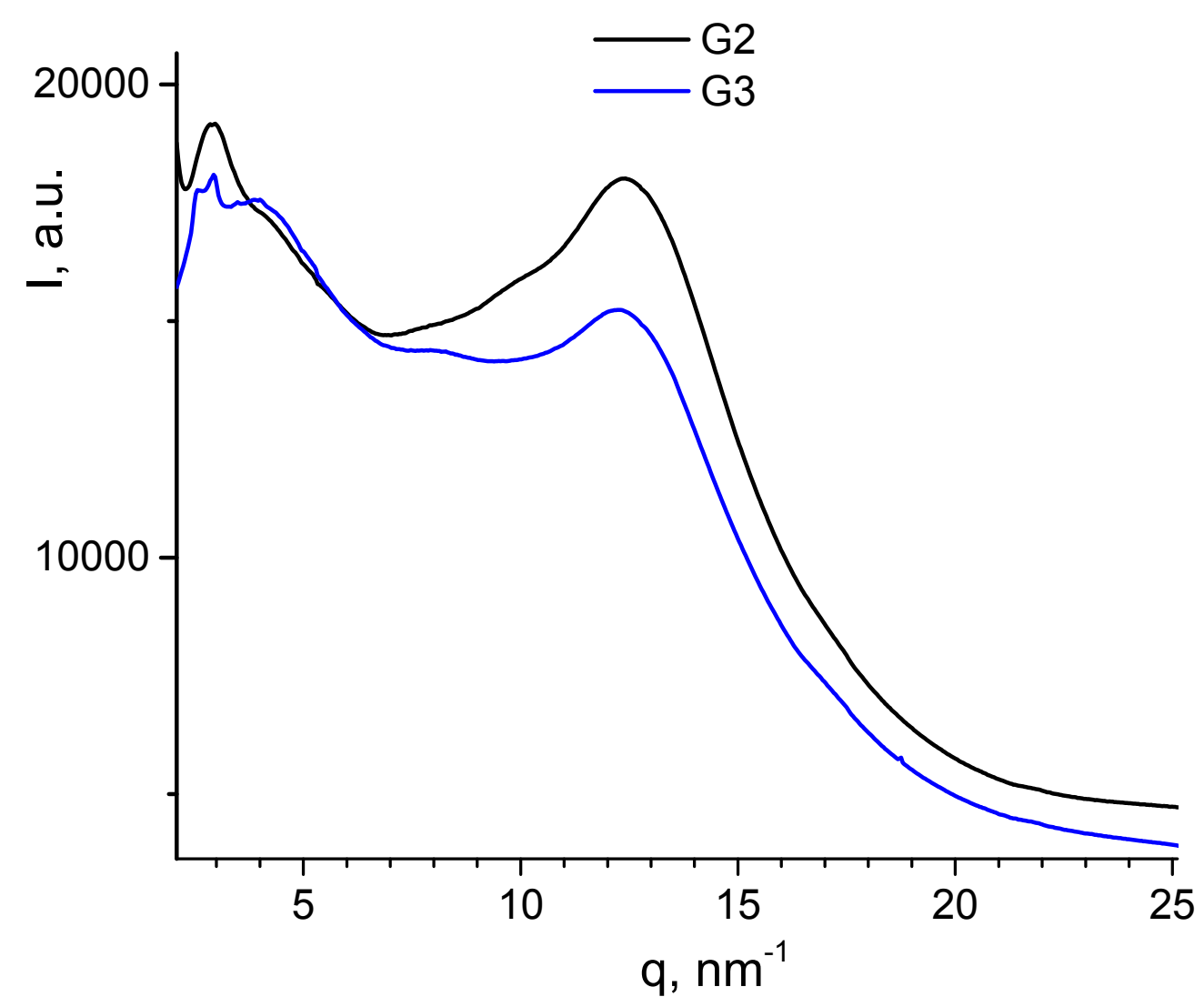

Figure S2. XXX WAXS curves for G2-G3 dendrimers. 


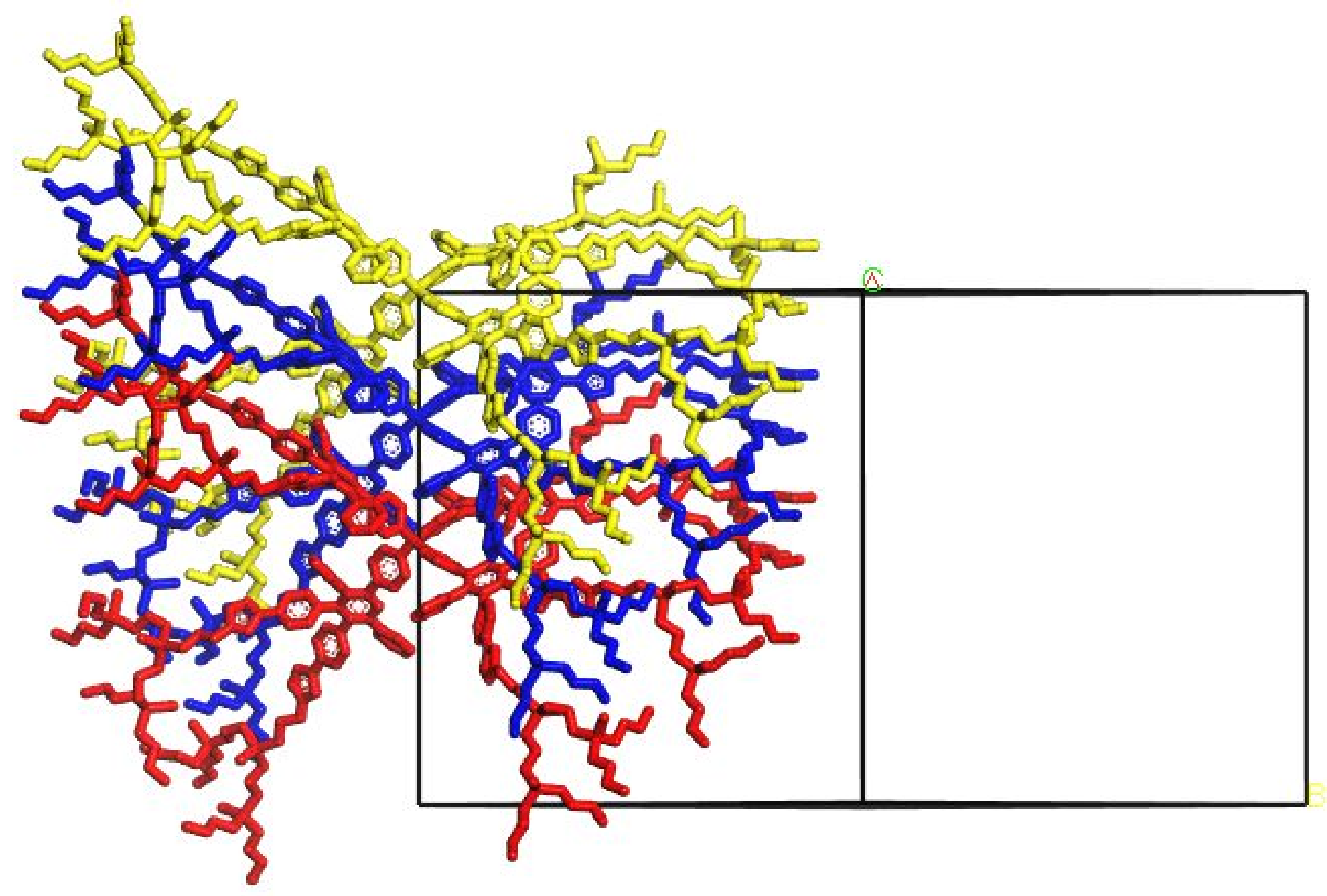

Figure S3. G1 Side View 


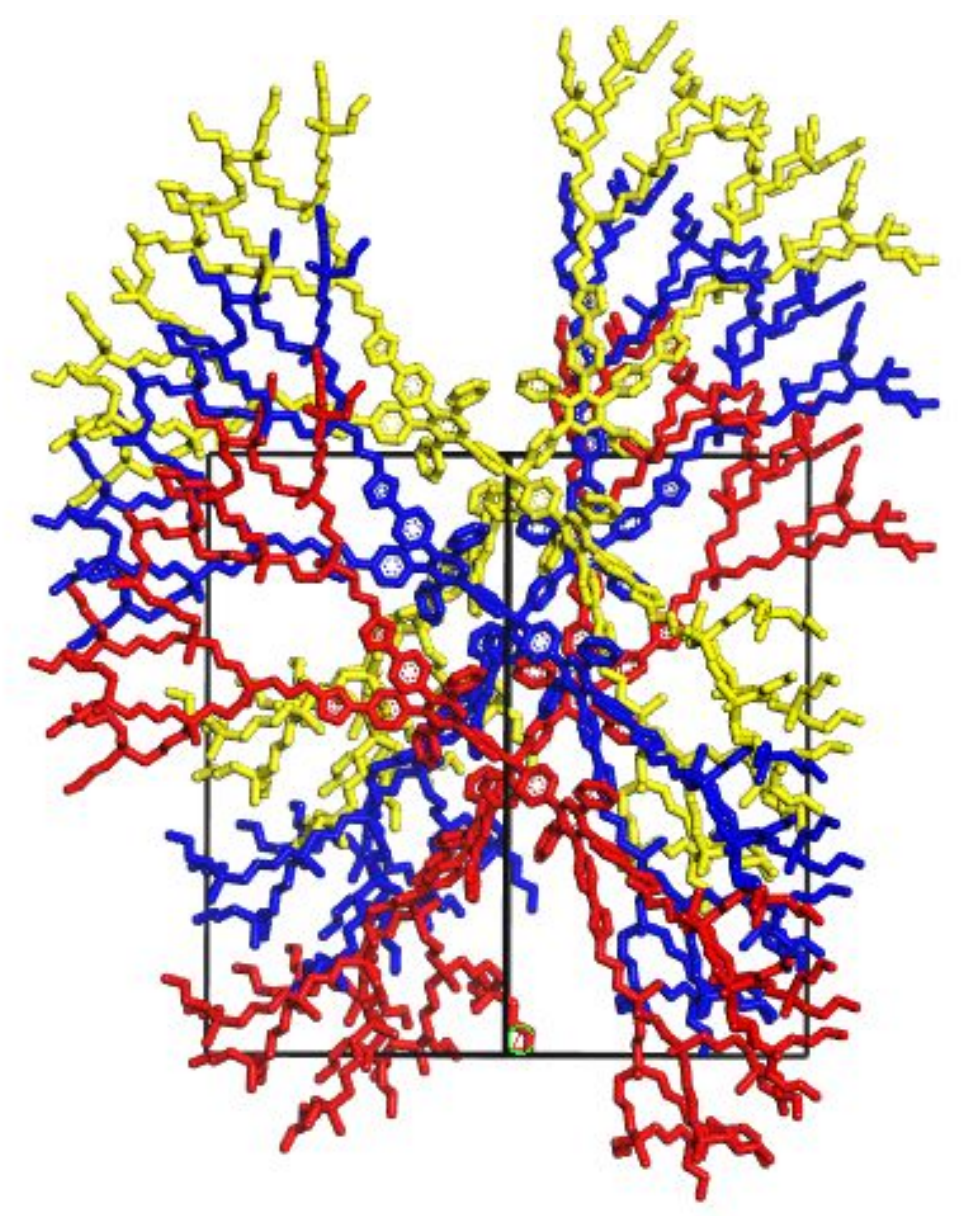

Figure S4. G2 Side View 


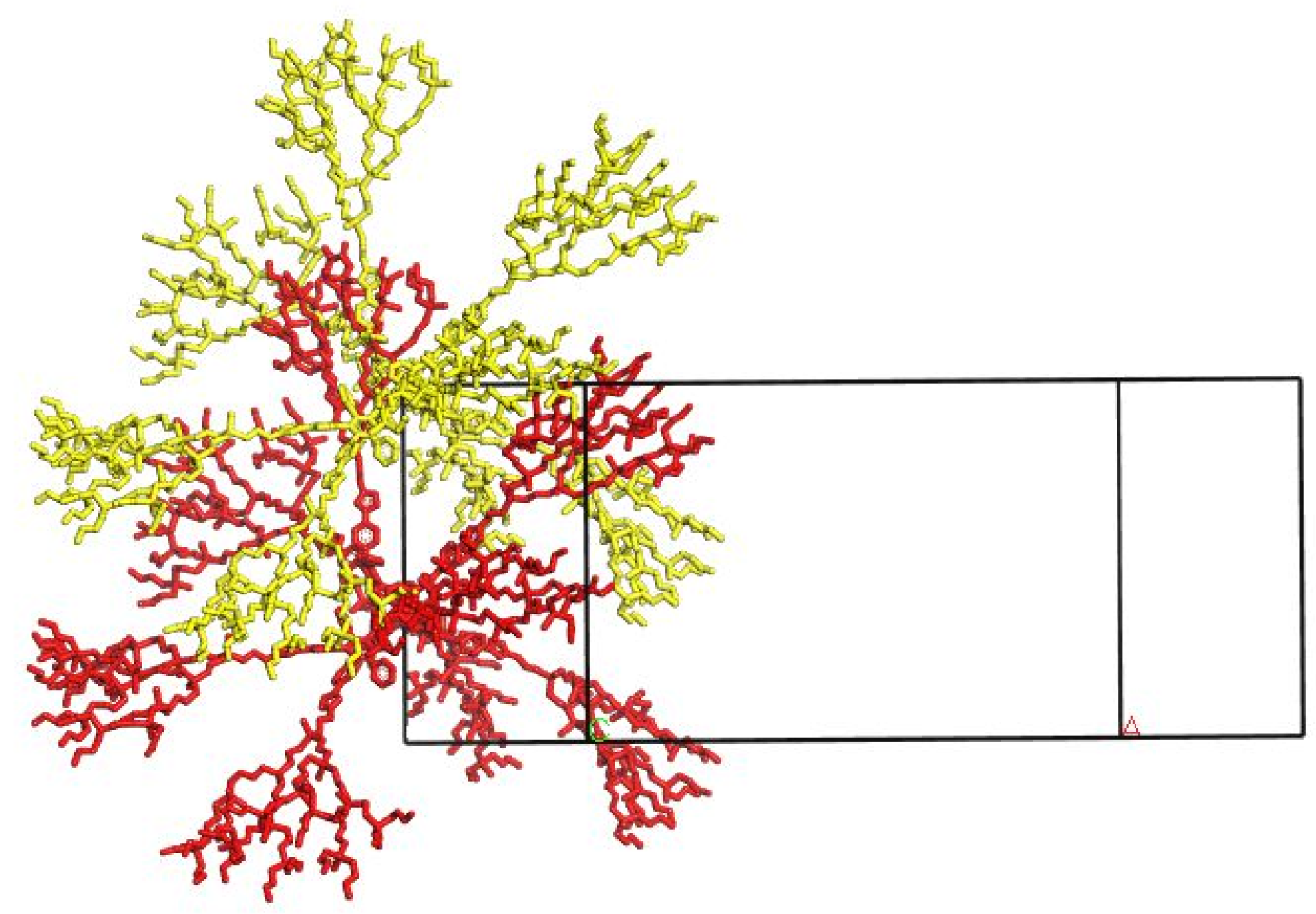

Figure S5. G2 Side View 


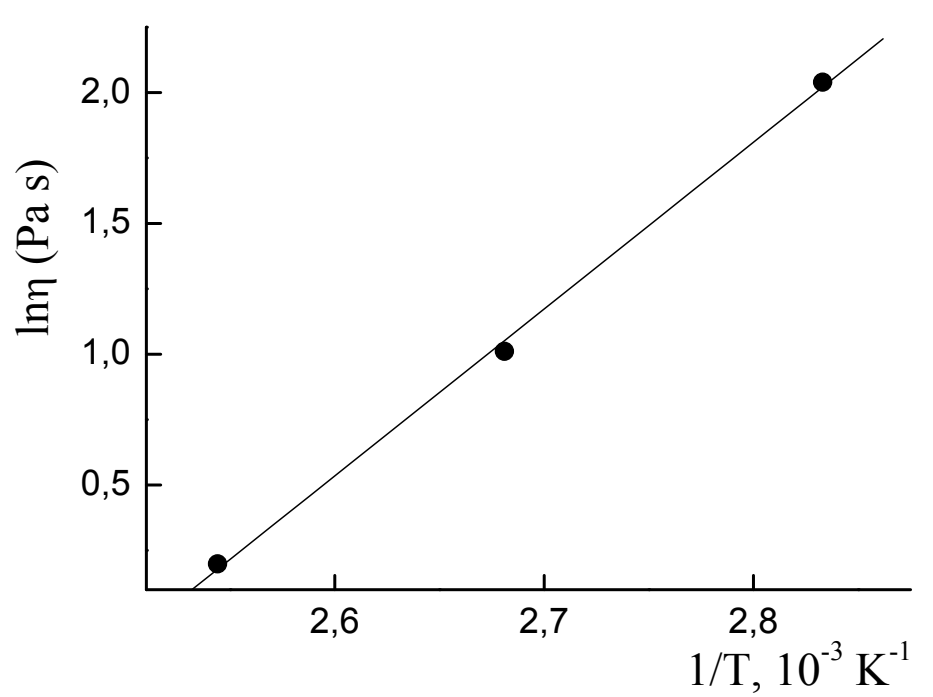

Figure S6. Viscosity dependence on the reverse temperature (in the coordinates of the Arrhenius equation). 